\title{
Facile Synthesis of Quaternary $\alpha$-Fluoronitriles by Cobalt-Catalyzed Hydrocyanation of Monofluoroalkenes
}

\author{
Yanlin Li \\ Ru Cui \\ Tian-Rui Wu \\ Xi-Sheng Wang*iD \\ Hefei National Laboratory for Physical Sciences at the \\ Microscale and Department of Chemistry, University of \\ Science and Technology of China, Hefei, Anhui 230026 , \\ P. R. of China \\ xswang77@ustc.edu.cn
}

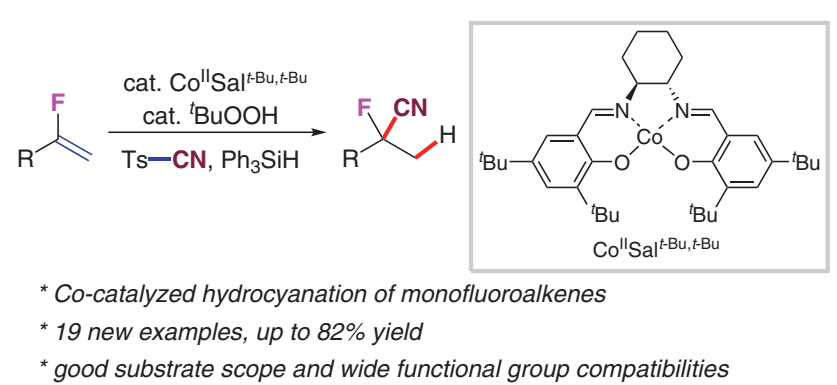

Received: 16.08 .2021

Accepted after revision: 07.09.2021

Published online: 21.10 .2021

DOI: 10.1055/s-0040-1719841; Art ID: ss-2021-g0492-op

Abstract An exclusively regioselective hydrocyanation of monofluoroalkenes has been developed, with which a series of aliphatic quaternary $\alpha$-fluoronitriles were synthesized in a facile and efficient manner. This novel method is featured with mild conditions, good functional groups compatibilities, and high reactivity.

Key words quaternary $\alpha$-fluoronitriles, hydrocyanation, monofluoroalkenes, cobalt catalysis, radical

The fluorine atom has a tiny atomic radius and the strongest electronegativity of the periodic table of elements. ${ }^{1}$ These natural characters make the introduction of fluorine into organic structure dramatically change its properties, such as metabolic stability, lipophilicity, bioavailability, and binding affinity. ${ }^{2}$ Meanwhile, nitriles have been extensively used as one of the most versatile intermediates in organic synthesis, enabling diverse chemical transformations. As one of the alternative precursors of $\beta$ fluoroamines, which serves as the key moieties in bioactive and pharmaceutical compounds, ${ }^{3}$ the efficient synthesis of $\alpha$-fluoronitriles have inspired wide attentions of organofluorine chemists. Despite the importance of $\alpha$-fluoronitriles, there are only few synthetic methods reported so far, especially for the quaternary $\alpha$-fluoronitriles. The common method to afford quaternary $\alpha$-fluoronitriles is direct fluorination by the construction of $\mathrm{C}-\mathrm{F}$ bonds, via either dehydroxylate fluorination of cyanohydrins ${ }^{4}$ (Scheme 1, Path I) or electrophilic fluorination of in situ generated carbanion ${ }^{5}$ (Scheme 1, Path II). However, the instability of cyanohydrins for the dehydroxylate fluorination and the requirement of electron-drawing group (EWG) adjacent to the cyano group for the electrophilic fluorination definitely hampered their utility for synthetic applications. Thus, an alternative method has been developed by $\mathrm{C}-\mathrm{C}$ bond formation via nucleophilic addition of tertiary $\alpha$-fluoronitrile carbanion to electrophiles ${ }^{6}$ (Scheme 1, Path III-IV), in which the reaction types and substrate scope was correspondingly limited. Indeed, the diverse construction of quaternary $\alpha-$ fluoronitriles, especially bearing no other activating group on the quaternary carbon center, remains still an unsolved issue to be addressed.

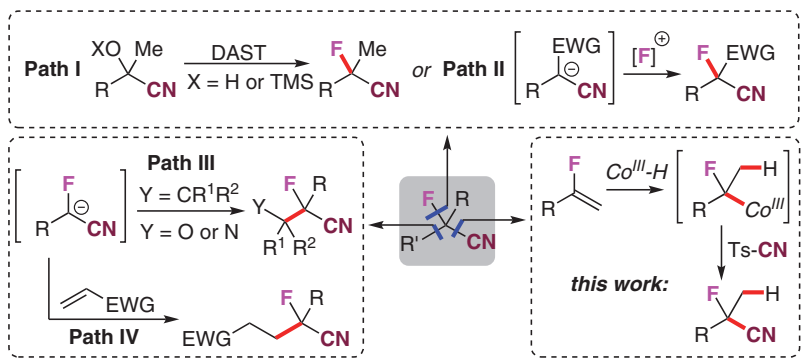

Scheme 1 Synthesis of quaternary $\alpha$-fluoronitriles

As we are very much inspired by the exploration for facile synthetic methods for the various monofluorinated compounds with new molecular platforms, the construction of quaternary $\alpha$-fluoronitrile has accordingly aroused our research interests. ${ }^{7}$ Considering that alkenes served normally as one kind of simple and basal materials for further transformation to diverse complex molecules, we envisioned that monofluoroalkenes may play as a potential molecular platform to construct various monofluorine-containing compounds. ${ }^{8}$ As is known, the metal-hydride hydrogen atom transfer (MHAT) process is used as a strong strategy for the hydrofunctionalization of alkenes by an in situ generation of hydrogenated carbon radical or metal species, followed by various radical captures or metal-catalyzed functionalizations. ${ }^{9}$ Accordingly, we speculated that a 
$\mathrm{Co}^{\mathrm{III}}$-H-promoted radical hydrocyanation of monofluoroalkenes would pave a new approach for effective construction of quaternary $\alpha$-fluoronitriles, by a strategical cleavage of $\mathrm{C}-\mathrm{CN}$ bonds. Herein, we describe an exclusively regioselective hydrocyanation of monofluoroalkenes, with which a series of aliphatic quaternary $\alpha$-fluoronitriles were synthesized in a facile and efficient manner. This novel method is featured with mild conditions, well functional groups compatibilities, and high reactivity.

At the beginning, our study commenced with methyl 2-(2-fluoroallyl)isoindoline-1,3-dione (1) as the initial substrate, tosyl cyanide $(0.15 \mathrm{mmol}, 1.5$ equiv) as the cyano source, and $\mathrm{PhSiH}_{3}$ as the hydride source in the presence of a catalytic amount of $\mathrm{Co}^{\mathrm{II} S a l} \mathrm{Sa}^{t-B u, t-B u}(10 \mathrm{~mol} \%)$ in EtOH. To our delight, the desired quaternary $\alpha$-fluoronitrile $\mathbf{2}$ was obtained smoothly in $47 \%$ yield when 0.3 equivalent of $t$-BuOOt-Bu was added to the reaction as oxidant to gener- ate the active catalyst (Table 1 , entry 1 ). Considering this metal-hydride hydrogen atom transfer (MHAT) process was initiated only by $\mathrm{Co}^{\mathrm{III}}-\mathrm{H}$ species, different kinds of oxidants were examined under such reaction conditions at first (entries 2-6). The resulting data show that a number of added oxidants, including $t$-BuOOH, $\mathrm{PhCO}_{2} \mathrm{Ot}$-Bu, Selectfluor, NFSI, and $\mathrm{BIOH}$, could start this radical reaction and provide the desired product $\mathbf{2}$ in tolerable yields, but $t-\mathrm{BuOOH}$ favored the hydrocyanation of monofluoroalkene with a slightly higher yield (entry 2). In order to adjust the rate of $\mathrm{Co}-\mathrm{H}$ species generation to match the rate of radical capture, various $\mathrm{Si}-\mathrm{H}$ reagents, including, activated $\mathrm{HSi}(\mathrm{OEt})_{3}$, $\mathrm{HSiMe}(\mathrm{OEt})_{2}$, and PMHS or non-activated PhSiMeH and $\mathrm{Ph}_{2} \mathrm{SiH}_{2}$ were carefully investigated in this cobalt catalytic system (entries 7-11). The results revealed that the nonactivated $\mathrm{Si}-\mathrm{H}$ reagents adapted this reaction conditions better, and $\mathrm{PhSiH}_{3}$ still gave the best yield of aliphatic qua-

Table 1 Optimization of the Reaction Conditions ${ }^{\mathrm{a}}$

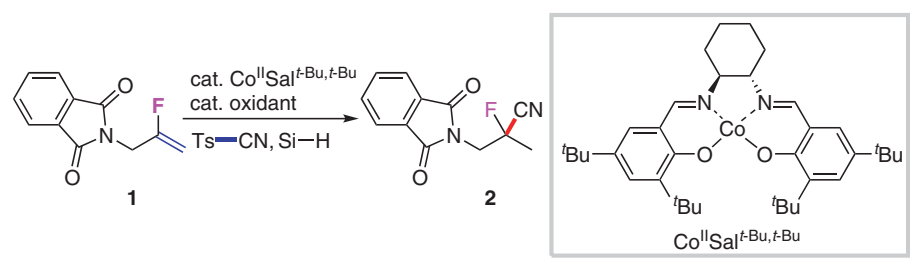

\begin{tabular}{|c|c|c|c|c|}
\hline Entry & $\mathrm{Si}-\mathrm{H}$ & Oxidant & Solvent $(\mathrm{mL})$ & Yield (\%) \\
\hline 1 & $\mathrm{PhSiH}_{3}$ & $t$-BuOOt-Bu & $\mathrm{EtOH}$ & 47 \\
\hline 2 & $\mathrm{PhSiH}_{3}$ & $t-\mathrm{BuOOH}$ & $\mathrm{EtOH}$ & 49 \\
\hline 3 & $\mathrm{PhSiH}_{3}$ & $\mathrm{PhCO}_{2} \mathrm{Ot}-\mathrm{Bu}$ & $\mathrm{EtOH}$ & 41 \\
\hline 4 & $\mathrm{PhSiH}_{3}$ & Selectfluor & $\mathrm{EtOH}$ & 37 \\
\hline 5 & $\mathrm{PhSiH}_{3}$ & NFSI & $\mathrm{EtOH}$ & 32 \\
\hline 6 & $\mathrm{PhSiH}_{3}$ & $\mathrm{BIOH}$ & $\mathrm{EtOH}$ & 45 \\
\hline 7 & $\mathrm{HSi}(\mathrm{OEt})_{3}$ & $t$-BuOOH & $\mathrm{EtOH}$ & 2 \\
\hline 8 & $\mathrm{HSiMe}(\mathrm{OEt})_{2}$ & $t$-BuOOH & $\mathrm{EtOH}$ & 9 \\
\hline 9 & PMHS & $t-\mathrm{BuOOH}$ & $\mathrm{EtOH}$ & 6 \\
\hline 10 & $\mathrm{PhSiMeH}_{2}$ & $t-\mathrm{BuOOH}$ & $\mathrm{EtOH}$ & 45 \\
\hline 11 & $\mathrm{Ph}_{2} \mathrm{SiH}_{2}$ & $t-\mathrm{BuOOH}$ & $\mathrm{EtOH}$ & 45 \\
\hline 12 & $\mathrm{PhSiH}_{3}$ & $t-\mathrm{BuOOH}$ & acetone $(0.9) / \mathrm{EtOH}(0.1)$ & 34 \\
\hline 13 & $\mathrm{PhSiH}_{3}$ & $t-\mathrm{BuOOH}$ & $\mathrm{DCE}(0.9) / \mathrm{EtOH}(0.1)$ & 45 \\
\hline 14 & $\mathrm{PhSiH}_{3}$ & $t-\mathrm{BuOOH}$ & DME (0.9)/EtOH (0.1) & 38 \\
\hline 15 & $\mathrm{PhSiH}_{3}$ & $t-\mathrm{BuOOH}$ & $\operatorname{MeCN}(0.9) / \operatorname{EtOH}(0.1)$ & 15 \\
\hline $16^{\mathrm{b}}$ & $\mathrm{PhSiH}_{3}$ & $t-\mathrm{BuOOH}$ & $\mathrm{EtOH}$ & 34 \\
\hline $17^{b, c}$ & $\mathrm{PhSiH}_{3}$ & $t-\mathrm{BuOOH}$ & $\mathrm{EtOH}$ & 60 \\
\hline $18^{\mathrm{b}, \mathrm{d}}$ & $\mathrm{PhSiH}_{3}$ & $t-\mathrm{BuOOH}$ & $\mathrm{EtOH}$ & $84(82)$ \\
\hline
\end{tabular}

a Reaction conditions: 1 ( 0.1 mmol, 1.0 equiv), TsCN (1.5 equiv), Co"Salt-Bu,t-Bu $(10 \mathrm{~mol} \%)$, Si-H (1.0 equiv), oxidant ( 0.3 equiv), solvent (1 mL), r.t., 12 h. Yields were determined by ${ }^{19} \mathrm{~F}$ NMR spectroscopy using $\mathrm{PhCF}_{3}$ as an internal standard; numbers in parentheses were yields of isolated products. PMHS = Poly(methylhydrosiloxane).

b TsCN (3.0 equiv) was used.

c $\mathrm{PhSiH}_{3}$ (1.5 equiv) was used.

${ }^{d} \mathrm{PhSiH}_{3}$ (2.5 equiv) was used. 
ternary $\alpha$-fluoronitrile 2 . Meanwhile, the optimization of solvents indicated mixed solvents were unhelpful for this reaction (entries 12-15), and only DCM (0.9 mL)/EtOH (0.1 $\mathrm{mL}$ ) gave a similar yield as in entry 2 . While increasing the loading of TsCN to $0.3 \mathrm{mmol}$ slightly decreased the yield (entry 16), to our satisfaction, the enhancement of the equivalent of both $\mathrm{Ts}-\mathrm{CN}(0.3 \mathrm{mmol})$ and $\mathrm{PhSiH}_{3}(0.15$ mmol) could clearly improve the yield of target product to $60 \%$ (entry 17). This result indicated that the loading of $\mathrm{Si}-\mathrm{H}$ reagent should be closely related to $\mathrm{Ts}-\mathrm{CN}$ and was crucial for the transformation. Finally, further increasement of the loading of $\mathrm{PhSiH}_{3}$ to 2.5 equivalents furnished the aliphatic quaternary $\alpha$-fluoronitriles 2 in $82 \%$ isolated yield (entry 18).

With the optimized reaction conditions in hand, we next explored the compatibilities with various monofluoroalkenes in this cobalt catalytic system (Scheme 2). As expected, the monofluoroalkenes with a longer carbon chain could adapt this radical reaction well affording 4, which indicated that a directing group was not necessary for this transformation. Furthermore, the monofluoroalkenes installed with benzamide group were also compatible with the optimized conditions, affording the desired products $\mathbf{2}$ and $\mathbf{5}$ in acceptable to good yields. Meanwhile, the benzoate-derived monofluoroalkenes were also suitable substrates for this transformation $(\mathbf{6}-\mathbf{1 5}, \mathbf{1 8})$. Notably, diverse monofluoroalkenes containing different phenyl rings which were equipped with various functional groups, such as methyl (7), isopropyl (8), methoxy (9), phenyl (10), ester (12), cyano (13), and trifluoromethyl $(\mathbf{1 1}, \mathbf{1 4})$, could all provide the corresponding products in moderate to good yields. To our interests, the substrates installed with orthofunctional groups on the phenyl rings, no matter methoxy (15) or bromo (18), could also furnish the desired quaternary $\alpha$-fluoronitrile smoothly, albeit in slightly decreased yields. Inspired by these interesting results, this novel transformation has also been explored for late-stage modification of complex bioactive molecules. Accordingly, several monofluoroalkenes containing diversified pharmaceutical structures have been synthesized and subjected into the

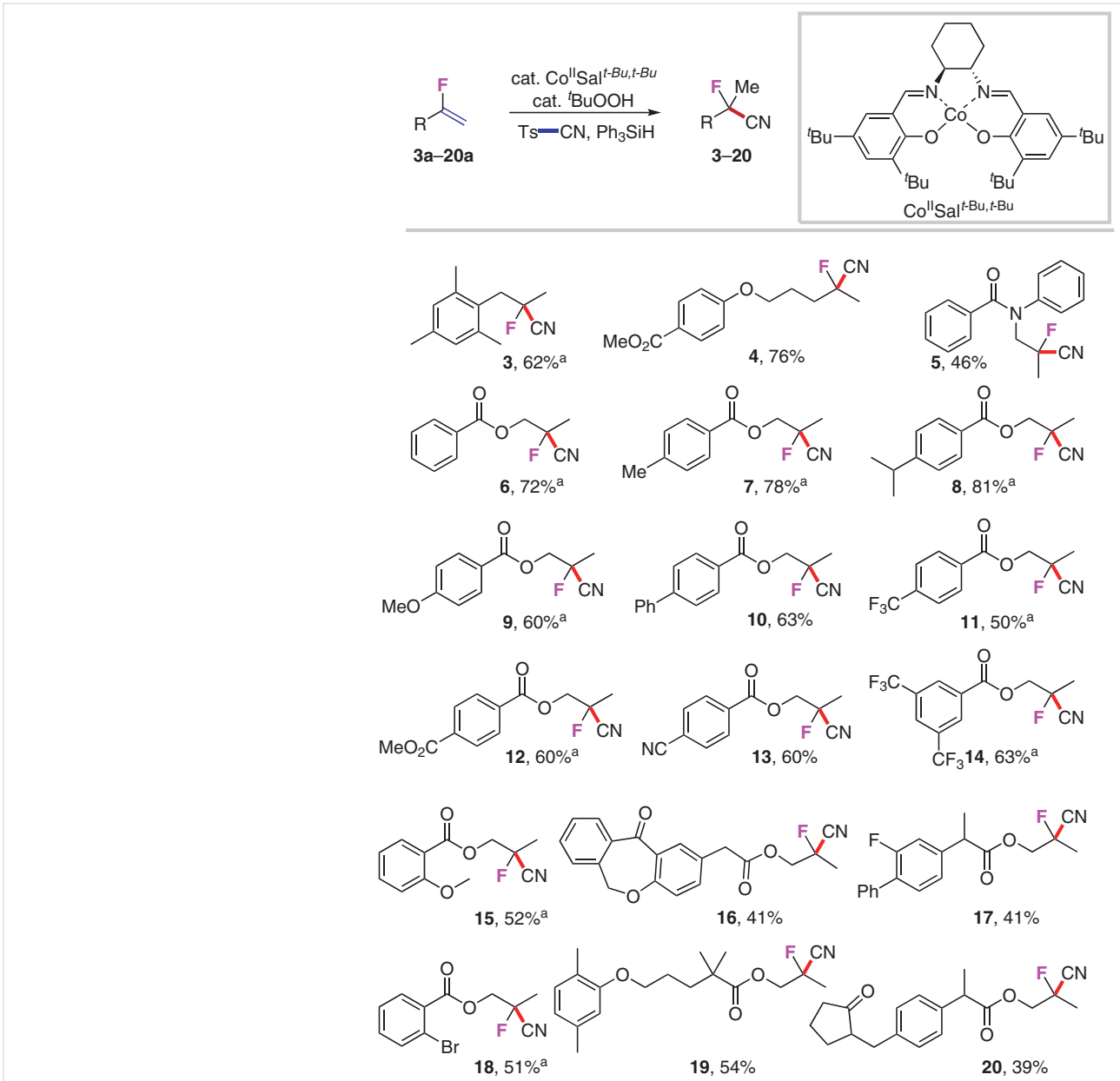

Scheme 2 Scope of monofluoroalkenes. Reagents and conditions: monofluoroalkene ( 0.1 mmol, 1.0 equiv), TsCN (3.0 equiv), Co"Saltt-Bu,t-Bu (10 mol\%),

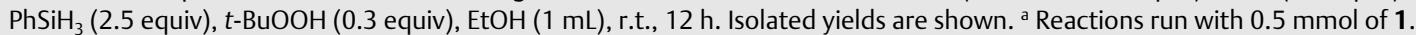


hydrocyanation conditions. To our excitement, such monofluoroalkenes derived from diverse drugs, such as isoxepac (16), flurbiprofen (17), gemfibrozil (19), and loxoprofen (20), all were compatible well with this transformation in acceptable yields.

In order to confirm the hydride source of this hydrocyanation reaction, $\mathrm{PhSiD}_{3}$ has been used as hydride source to subject into the standard conditions by replacement of $\mathrm{PhSiH}_{3}$, affording the corresponding product 2-D in $62 \%$ yield with 99\% deuterium incorporation (Scheme 3). This result clearly indicated that the hydrogen atom for hydrocyanation comes from the $\mathrm{Si}-\mathrm{H}$ species via a MHAT process.

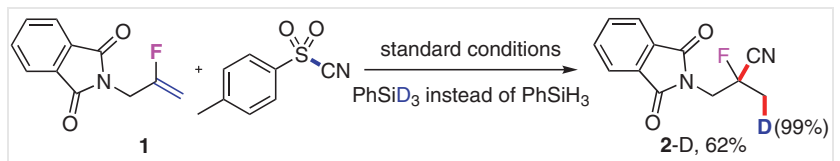

Scheme 3 Deuterium experiment

Based on previous reports ${ }^{9}$ and the above deuterium experiment result, a possible mechanism is proposed as shown in Scheme 4. Initially, the $\mathrm{Co}^{\mathrm{II}}$ species is oxidized to the active $\mathrm{Co}^{\mathrm{III}}$ species, ${ }^{9 \mathrm{i}-\mathrm{k}}$ which generates the $\mathrm{Co}^{\mathrm{III}}-\mathrm{H}$ species by interaction with $\mathrm{PhSiH}_{3}$. Subsequently, the insertion of monofluoroalkene into the $\mathrm{Co}^{\text {III }}-\mathrm{H}$ bond affords alkylated cobalt species $\mathbf{A}$. The following homolytic cleavage of $\mathrm{C}-\mathrm{Co}^{\mathrm{III}}$ bond provides carbon radical $\mathbf{B}$, followed by a radical capture by TsCN to furnish the final product $\mathbf{2}$ and produces Ts ${ }^{*}$ radical. Finally, the $\mathrm{Ts}^{\bullet}$ ( or $t-\mathrm{BuOOH}$ ) oxidizes $\mathrm{Co}^{\mathrm{II}}$ species to $\mathrm{Co}^{\mathrm{III}}$ species and completes the catalytic cycle.

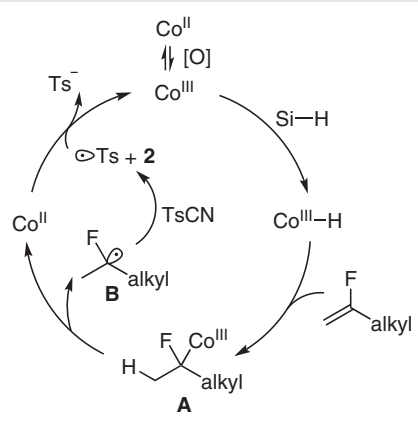

Scheme 4 Proposed mechanism

In summary, we have reported an exclusively regioselective hydrocyanation of monofluoroalkenes. This method paves a novel way to construct a series of aliphatic quaternary $\alpha$-fluoronitriles, and featured with mild conditions, good functional groups compatibilities, and high reactivity. Further explorations for regio- and stereoselective construction of monofluorine-containing quaternary carbon center are underway in our laboratory.
Chemical shifts were reported in ppm from the solvent resonance as the internal standard $\left(\mathrm{CDCl}_{3} \delta_{\mathrm{H}}=7.26, \delta_{\mathrm{C}}=77.16\right.$. Standard abbreviations were used to indicate multiplicities. Coupling constants were reported in hertz $(\mathrm{Hz})$. High-resolution mass spectra were recorded on P-SIMS-Gly of Bruker Daltonics Inc. using ESI-TOF (electrospray ionization-time of flight). The monofluoroalkenes were synthesized according to following methods. Anhydrous solvents and commercially available reagents were purchased and used without further purification. Flash column chromatography was carried out using silica gel (200-300 mesh) with the indicated solvent system. All reactions were conducted in oven-dried Schlenk tubes.

\section{2-(2-Fluoroallyl)isoindoline-1,3-dione (1); Typical Procedure 1 (TP 1)}

The starting material 2-fluoroprop-2-en-1-ol was synthesized according to a reported procedure ${ }^{10}$ from methyl 2-fluoroacrylate $(5.2 \mathrm{~g}$, $50 \mathrm{mmol}$ ). After removing the solvent carefully, the crude 2-fluoroprop-2-en-1-ol was dissolved in THF $(0.5 \mathrm{M})$. At $0{ }^{\circ} \mathrm{C}$, to the solution was added $\mathrm{NaH}$ (60\% in mineral oil, 1.2 equiv) slowly and the mixture was stirred for $5 \mathrm{~min}$. Then $\mathrm{TsCl}$ (1.1 equiv) in THF was added dropwise and the reaction was stirred overnight. $\mathrm{H}_{2} \mathrm{O}(100 \mathrm{~mL})$ and EtOAc $(100 \mathrm{~mL})$ were added to the reaction mixture, then the aqueous phase was extracted with EtOAc $(3 \times 100 \mathrm{~mL})$ and the organic layers were combined, washed with brine, and dried $\left(\mathrm{Na}_{2} \mathrm{SO}_{4}\right)$. The organic layer was concentrated for flash column chromatography on silica gel with an eluent of PE and EtOAc (10:1) to obtain the crude 2-furoprop-2enyl toslate. Next, phthalimide $(7.36 \mathrm{~g}, 50 \mathrm{mmol})$ was dissolved in THF, followed by the addition of $\mathrm{NaH}$ ( $60 \%$ in mineral oil, 1.2 equiv) at $0{ }^{\circ} \mathrm{C}$. After stirring for $5 \mathrm{~min}$, the tosylate from the last step was added to the reaction mixture and allowed to stay overnight at r.t. After quenching with $\mathrm{H}_{2} \mathrm{O}(100 \mathrm{~mL})$, EtOAc $(100 \mathrm{~mL})$ was added to the mixture, and the aqueous phase was extracted with EtOAc $(3 \times 100 \mathrm{~mL})$. The organic layers were combined, washed with brine, and dried $\left(\mathrm{Na}_{2} \mathrm{SO}_{4}\right)$. The mixture was concentrated for column chromatography on silica gel with an eluent of PE and EtOAc to obtain the final product 1; total yield: $3.6 \mathrm{~g}(35 \%)$.

Similarly, 5a was prepared from $\mathrm{N}$-phenylbenzamide and 2-fluoroprop-2-en-1-ol.

\section{2-Fluoroallyl Benzoate (6a); Typical Procedure 2 (TP 2)}

The starting material 2-fluoroprop-2-en-1-ol was synthesized according to a reported procedure ${ }^{10}$ from methyl 2-fluoroacrylate (5.2.g, $50 \mathrm{mmol})$, and then the crude 2-fluoroprop-2-en-1-ol was stirred with PhCOCl (7.03 g, $50 \mathrm{mmol}, 1.0$ equiv) in $\mathrm{CH}_{2} \mathrm{Cl}_{2}(0.5 \mathrm{M})$ at $0{ }^{\circ} \mathrm{C}$. $\mathrm{NEt}_{3}$ (10.1 g, $100 \mathrm{mmol}, 2$ equiv) was added to the reaction mixture and the mixture was stirred overnight. After total consumption of the starting material, the mixture was concentrated under vacuum. The residue was then purified by flash column chromatography (PE/EtOAc 10:1) to give the target product 6a; yield: $6.5 \mathrm{~g}$ (72\%).

Vinyl fluorides 7a-20a were prepared from suitable starting materials based on the above typical procedure 2 .

\section{2-(2-Fluoroallyl)-1,3,5-trimethylbenzene (3a)}

2,4,6-Trimethylphenylmagnesium bromide (1 M in THF, $2.23 \mathrm{~g}, 10$ mmol, 1 equiv) was stirred at r.t. Anhyd THF ( $30 \mathrm{~mL})$ and 2-fluoroallyl 4-methylbenzenesulfonate ( $2.3 \mathrm{~g}, 10 \mathrm{mmol}, 1$ equiv) were added sequentially to the reaction mixture. The mixture was stirred at $60{ }^{\circ} \mathrm{C}$ for $4 \mathrm{~h}$. Afterwards, the mixture was quenched with aq $1 \mathrm{M} \mathrm{HCl}$. The organic phase was washed with aq $1 \mathrm{M} \mathrm{HCl}$, and the aqueous phase was extracted with EtOAc $(3 \times 50 \mathrm{~mL})$. The organic layers were com- 
bined, and concentrated under vacuum. The residue was purified by flash column chromatography (PE) to give the target product 3a; yield: $540 \mathrm{mg}$ (30\%).

\section{2-(2-Fluoroallyl)isoindoline-1,3-dione (1)}

Purified by silica gel chromatography (PE/EtOAc 5:1); white solid; mp $82.8-84.5^{\circ} \mathrm{C}$.

${ }^{1} \mathrm{H}$ NMR $\left(500 \mathrm{MHz}, \mathrm{CDCl}_{3}\right): \delta=7.87(\mathrm{dd}, J=5.4,3.1 \mathrm{~Hz}, 2 \mathrm{H}), 7.74(\mathrm{dd}$, $J=5.5,3.0 \mathrm{~Hz}, 2 \mathrm{H}$ ), 4.75 (dd, $J=16.1,3.5 \mathrm{~Hz}, 1 \mathrm{H}$ ), 4.56 (dd, $J=47.6$, $3.4 \mathrm{~Hz}, 1 \mathrm{H}), 4.39$ (d, J= $12.1 \mathrm{~Hz}, 2 \mathrm{H})$.

${ }^{13} \mathrm{C}$ NMR (126 MHz, $\mathrm{CDCl}_{3}$ ): $\delta=167.50,159.68(\mathrm{~d}, J=260.0 \mathrm{~Hz})$, 134.37, 132.00, 123.69, $93.06(\mathrm{~d}, J=17.1 \mathrm{~Hz}), 37.99(\mathrm{~d}, J=34.8 \mathrm{~Hz})$.

${ }^{19} \mathrm{~F} \mathrm{NMR} \mathrm{(376} \mathrm{MHz,} \mathrm{CDCl}_{3}$ ): $\delta=-103.71$ (ddt, $J=48.2,15.3,12.2 \mathrm{~Hz}$ ). HRMS (ESI): $m / z$ [M + Na+] calcd for $\mathrm{C}_{11} \mathrm{H}_{8} \mathrm{FNO}_{2} \mathrm{Na}^{+}: 228.0431$; found: 228.0427.

\section{2-(2-Fluoroallyl)-1,3,5-trimethylbenzene (3a)}

Purified by silica gel chromatography (PE); colorless oil.

${ }^{1} \mathrm{H}$ NMR $\left(400 \mathrm{MHz}, \mathrm{CDCl}_{3}\right): \delta=6.92(\mathrm{~s}, 2 \mathrm{H}), 4.65-4.49(\mathrm{~m}, 1 \mathrm{H}), 4.07-$ $3.82(\mathrm{~m}, 1 \mathrm{H}), 3.54(\mathrm{~d}, J=6.7 \mathrm{~Hz}, 2 \mathrm{H}), 2.35(\mathrm{~s}, 6 \mathrm{H}), 2.33(\mathrm{~s}, 3 \mathrm{H})$.

${ }^{13} \mathrm{C}$ NMR $\left(126 \mathrm{MHz}, \mathrm{CDCl}_{3}\right): \delta=164.82(\mathrm{~d}, J=259.2 \mathrm{~Hz}), 137.15$, 136.46, 129.70 (d, $J=8.8 \mathrm{~Hz}), 129.11,89.84(\mathrm{~d}, J=19.5 \mathrm{~Hz}), 31.89$ (d, $J=29.2 \mathrm{~Hz}), 20.99,19.79$.

${ }^{19} \mathrm{~F} \operatorname{NMR}\left(376 \mathrm{MHz}, \mathrm{CDCl}_{3}\right.$ ): $\delta=-93.03$ (ddt, $J=50.1,17.3,6.7 \mathrm{~Hz}$ ). HRMS (EI): $m / z\left[\mathrm{M}^{+}\right]$calcd for $\mathrm{C}_{12} \mathrm{H}_{15} \mathrm{~F}^{+}$: 178.1152; found: 178.1152 .

\section{Methyl 4-[(4-Fluoropent-4-en-1-yl)oxy]benzoate (4a) $)^{11}$}

Purified by silica gel chromatography (PE/EtOAc 10:1); colorless oil.

${ }^{1} \mathrm{H} \mathrm{NMR}\left(500 \mathrm{MHz}, \mathrm{CDCl}_{3}\right): \delta=7.98(\mathrm{~d}, J=8.8 \mathrm{~Hz}, 2 \mathrm{H}), 6.90(\mathrm{~d}, J=8.8$ $\mathrm{Hz}, 2 \mathrm{H}), 4.56$ (dd, $J=17.4,2.8 \mathrm{~Hz}, 1 \mathrm{H}), 4.27(\mathrm{dd}, J=50.0,2.7 \mathrm{~Hz}, 1 \mathrm{H})$, $4.05(\mathrm{t}, J=6.1 \mathrm{~Hz}, 2 \mathrm{H}), 3.88(\mathrm{~s}, 3 \mathrm{H}), 2.48-2.30(\mathrm{~m}, 2 \mathrm{H}), 2.02(\mathrm{p}, J=6.4$ $\mathrm{Hz}, 2 \mathrm{H})$.

${ }^{13} \mathrm{C}$ NMR $\left(126 \mathrm{MHz}, \mathrm{CDCl}_{3}\right): \delta=167.00,165.79(\mathrm{~d}, J=257.1 \mathrm{~Hz})$, 162.78, 131.74, 122.78, 114.18, 90.51 (d, $J=20.2 \mathrm{~Hz}$ ), 66.80, 52.00, $28.60(\mathrm{~d}, J=28.0 \mathrm{~Hz}), 25.81$.

${ }^{19} \mathrm{~F} \mathrm{NMR}\left(471 \mathrm{MHz}, \mathrm{CDCl}_{3}\right): \delta=-95.57(\mathrm{dq}, J=50.4,16.8 \mathrm{~Hz})$.

HRMS (ESI): $m / z\left[\mathrm{M}+\mathrm{H}^{+}\right]$calcd for $\mathrm{C}_{13} \mathrm{H}_{16} \mathrm{FO}_{3}{ }^{+}:$239.1078; found: 239.1083.

\section{$\boldsymbol{N}$-(2-Fluoroallyl)- $\boldsymbol{N}$-phenylbenzamide (5a)}

Purified by silica gel chromatography (PE/EtOAc 5:1); colorless oil. ${ }^{1} \mathrm{H} \mathrm{NMR}\left(500 \mathrm{MHz}, \mathrm{CDCl}_{3}\right): \delta=7.36-7.30(\mathrm{~m}, 2 \mathrm{H}), 7.25-7.19(\mathrm{~m}, 3 \mathrm{H})$, 7.19-7.12 (m, $3 \mathrm{H}), 7.08(\mathrm{~d}, J=7.6 \mathrm{~Hz}, 2 \mathrm{H}), 4.74(\mathrm{dd}, J=16.5,3.2 \mathrm{~Hz}, 1$ $\mathrm{H}), 4.64(\mathrm{~d}, J=13.3 \mathrm{~Hz}, 2 \mathrm{H}), 4.54(\mathrm{dd}, J=48.4,3.1 \mathrm{~Hz}, 1 \mathrm{H})$.

${ }^{13} \mathrm{C}$ NMR $\left(126 \mathrm{MHz}, \mathrm{CDCl}_{3}\right): \delta=170.64,161.31(\mathrm{~d}, J=260.1 \mathrm{~Hz})$, 143.32, 135.56, 130.02, 129.28, 128.89, 127.90, 127.57, 127.08, 93.51 (d, $J=18.0 \mathrm{~Hz}), 50.38(\mathrm{~d}, J=32.2 \mathrm{~Hz}$ ).

$\left.{ }^{19} \mathrm{~F} \mathrm{NMR} \mathrm{(471} \mathrm{MHz,} \mathrm{CDCl}_{3}\right): \delta=-99.39$ to $-113.03(\mathrm{~m})$.

HRMS (ESI): $m / z\left[\mathrm{M}+\mathrm{H}^{+}\right]$calcd for $\mathrm{C}_{16} \mathrm{H}_{15} \mathrm{FNO}^{+}$: 256.1132; found: 256.1140 .

\section{2-Fluoroallyl Benzoate (6a)}

Purified by silica gel chromatography (PE/EtOAc 30:1); colorless oil. $\left.{ }^{1} \mathrm{H} \mathrm{NMR} \mathrm{(400} \mathrm{MHz,} \mathrm{CDCl}_{3}\right): \delta=8.13-8.02(\mathrm{~m}, 2 \mathrm{H}), 7.60-7.53(\mathrm{~m}, 1 \mathrm{H})$, 7.49-7.39 (m, $2 \mathrm{H}), 4.86$ (dd, $J=15.9,3.3 \mathrm{~Hz}, 1 \mathrm{H}), 4.84(\mathrm{dd}, J=13.9$, $0.5 \mathrm{~Hz}, 2 \mathrm{H}), 4.71$ (dd, $J=47.3,3.3 \mathrm{~Hz}, 1 \mathrm{H})$.
${ }^{13} \mathrm{C}$ NMR $\left(101 \mathrm{MHz}, \mathrm{CDCl}_{3}\right): \delta=165.91,160.44(\mathrm{~d}, J=257.9 \mathrm{~Hz})$, 133.44, 129.8, 129.58, 128.57, $94.53(\mathrm{~d}, J=17.0 \mathrm{~Hz}), 61.80(\mathrm{~d}, J=34.1$ $\mathrm{Hz})$.

${ }^{19} \mathrm{~F}$ NMR $\left(376 \mathrm{MHz}, \mathrm{CDCl}_{3}\right): \delta=-105.33(\mathrm{ddt}, J=47.3,15.8,13.9 \mathrm{~Hz}$ ). HRMS (ESI): $m / z\left[\mathrm{M}+\mathrm{H}^{+}\right]$calcd for $\mathrm{C}_{10} \mathrm{H}_{10} \mathrm{FO}_{2}^{+}$: 181.0659; found: 181.0662 .

\section{2-Fluoroallyl 4-Methylbenzoate (7a)}

Purified by silica gel chromatography (PE/EtOAc 30:1); colorless oil. ${ }^{1} \mathrm{H} \mathrm{NMR}\left(400 \mathrm{MHz}, \mathrm{CDCl}_{3}\right): \delta=7.96(\mathrm{~d}, J=8.2 \mathrm{~Hz}, 2 \mathrm{H}), 7.24(\mathrm{~d}, J=8.0$ $\mathrm{Hz}, 2 \mathrm{H}), 4.86(\mathrm{dd}, J=15.9,3.3 \mathrm{~Hz}, 1 \mathrm{H}), 4.83(\mathrm{~d}, J=13.8 \mathrm{~Hz}, 2 \mathrm{H}), 4.71$ (dd, $J=47.4,3.2 \mathrm{~Hz}, 1 \mathrm{H}), 2.41(\mathrm{~s}, 3 \mathrm{H})$.

${ }^{13} \mathrm{C}$ NMR $\left(126 \mathrm{MHz}, \mathrm{CDCl}_{3}\right): \delta=165.94,160.55(\mathrm{~d}, J=258.1 \mathrm{~Hz})$, $144.17,129.89,129.25,126.80,94.33(\mathrm{~d}, J=17.0 \mathrm{~Hz}), 61.58(\mathrm{~d}, J=34.1$ $\mathrm{Hz}), 21.73$.

${ }^{19} \mathrm{~F}$ NMR (376 MHz, $\left.\mathrm{CDCl}_{3}\right): \delta=-105.30$ (ddt, $J=47.4,15.9,13.9 \mathrm{~Hz}$ ). HRMS (ESI): $m / z\left[\mathrm{M}+\mathrm{Na}^{+}\right.$] calcd for $\mathrm{C}_{11} \mathrm{H}_{11} \mathrm{FO}_{2} \mathrm{Na}^{+}$: 217.0635; found: 217.0652 .

\section{2-Fluoroallyl 4-Isopropylbenzoate (8a)}

Purified by silica gel chromatography (PE/EtOAc 30:1); colorless oil. ${ }^{1} \mathrm{H} \mathrm{NMR}\left(400 \mathrm{MHz}, \mathrm{CDCl}_{3}\right): \delta=8.01(\mathrm{~d}, J=8.4 \mathrm{~Hz}, 2 \mathrm{H}), 7.31(\mathrm{~d}, J=8.2$ $\mathrm{Hz}, 2 \mathrm{H}), 4.86(\mathrm{dd}, J=16.0,3.2 \mathrm{~Hz}, 1 \mathrm{H}), 4.84(\mathrm{~d}, J=13.7 \mathrm{~Hz}, 2 \mathrm{H}), 4.71$ (dd, $J=47.4,3.2 \mathrm{~Hz}, 1 \mathrm{H}$ ), 2.97 (hept, $J=6.9 \mathrm{~Hz}, 1 \mathrm{H}$ ), 1.27 (d, $J=6.9 \mathrm{~Hz}$, $6 \mathrm{H})$.

${ }^{13} \mathrm{C}$ NMR $\left(126 \mathrm{MHz}, \mathrm{CDCl}_{3}\right): \delta=165.93,160.58(\mathrm{~d}, J=258.1 \mathrm{~Hz})$, $154.93,130.06,127.16,126.68,94.28(\mathrm{~d}, J=16.9 \mathrm{~Hz}), 61.57(\mathrm{~d}, J=34.2$ $\mathrm{Hz}), 34.38,23.76$.

${ }^{19} \mathrm{~F}$ NMR (376 MHz, $\mathrm{CDCl}_{3}$ ): $\delta=-105.34$ (ddt, $J=47.3,15.8,13.7 \mathrm{~Hz}$ ). HRMS (ESI): $m / z\left[\mathrm{M}+\mathrm{H}^{+}\right]$calcd for $\mathrm{C}_{13} \mathrm{H}_{16} \mathrm{FO}_{2}{ }^{+}$: 223.1129; found: 223.1137.

\section{2-Fluoroallyl 4-Methoxybenzoate (9a)}

Purified by silica gel chromatography (PE/EtOAc 30:1); colorless oil. ${ }^{1} \mathrm{H} \mathrm{NMR}\left(400 \mathrm{MHz}, \mathrm{CDCl}_{3}\right): \delta=8.02(\mathrm{~d}, J=8.7 \mathrm{~Hz}, 2 \mathrm{H}), 6.92(\mathrm{~d}, J=8.8$ $\mathrm{Hz}, 2 \mathrm{H}$ ), 4.88-4.77 (m, $3 \mathrm{H}$ ), 4.69 (dd, $J=47.4,3.2 \mathrm{~Hz}, 1 \mathrm{H}$ ), 3.86 (s, 3 $\mathrm{H})$.

${ }^{13} \mathrm{C}$ NMR (126 MHz, $\left.\mathrm{CDCl}_{3}\right): \delta=165.69,163.81,160.68(\mathrm{~d}, J=257.7$ $\mathrm{Hz}), 131.99,121.96,113.85,94.32(\mathrm{~d}, J=17.3 \mathrm{~Hz}), 61.55$ (d, $J=34.5$ $\mathrm{Hz}), 55.58$.

${ }^{19} \mathrm{~F}$ NMR (471 MHz, $\mathrm{CDCl}_{3}$ ): $\delta=-105.33(\mathrm{dq}, J=46.9,14.5 \mathrm{~Hz}$ ). HRMS (ESI): $m / z\left[\mathrm{M}+\mathrm{Na}^{+}\right]$calcd for $\mathrm{C}_{11} \mathrm{H}_{11} \mathrm{FO}_{3} \mathrm{Na}^{+}: 233.0584$; found: 233.0591.

\section{2-Fluoroallyl [1,1'-Biphenyl]-4-carboxylate (10a)}

Purified by silica gel chromatography (PE/EtOAc 30:1); white solid; mp $43.8-45.4^{\circ} \mathrm{C}$.

${ }^{1} \mathrm{H} \mathrm{NMR}\left(400 \mathrm{MHz}, \mathrm{CDCl}_{3}\right): \delta=8.14(\mathrm{~d}, J=7.7 \mathrm{~Hz}, 2 \mathrm{H}), 7.68(\mathrm{~d}, J=7.7$ $\mathrm{Hz}, 2 \mathrm{H}), 7.63(\mathrm{~d}, J=7.6 \mathrm{~Hz}, 2 \mathrm{H}), 7.48(\mathrm{t}, J=7.4 \mathrm{~Hz}, 2 \mathrm{H}), 7.44-7.36(\mathrm{~m}$, $1 \mathrm{H}), 4.91-4.85(\mathrm{~m}, 3 \mathrm{H}), 4.74(\mathrm{dd}, J=47.3,2.8 \mathrm{~Hz}, 1 \mathrm{H})$.

${ }^{13} \mathrm{C}$ NMR (126 MHz, $\mathrm{CDCl}_{3}$ ): $\delta=165.89,160.53(\mathrm{~d}, J=257.9 \mathrm{~Hz}$ ), $146.26,140.06,130.47,129.10,128.40,128.35,127.45,127.30,94.59$ (d, $J=17.1 \mathrm{~Hz}), 61.87(\mathrm{~d}, J=34.2 \mathrm{~Hz})$.

${ }^{19} \mathrm{~F} \operatorname{NMR}\left(376 \mathrm{MHz}, \mathrm{CDCl}_{3}\right): \delta=-105.31$ (dq, $\left.J=48.9,14.9 \mathrm{~Hz}\right)$.

HRMS (EI): $m / z$ [M+] calcd for $\mathrm{C}_{16} \mathrm{H}_{13} \mathrm{FO}_{2}{ }^{+}:$256.0894; found: 256.0896 . 


\section{2-Fluoroallyl 4-(Trifluoromethyl)benzoate (11a)}

Purified by silica gel chromatography (PE/EtOAc 30:1); colorless oil. ${ }^{1} \mathrm{H}$ NMR $\left(400 \mathrm{MHz}, \mathrm{CDCl}_{3}\right): \delta=8.19(\mathrm{~d}, J=8.1 \mathrm{~Hz}, 2 \mathrm{H}), 7.73(\mathrm{~d}, J=8.2$ $\mathrm{Hz}, 2 \mathrm{H}$ ), 4.90 (dd, $J=15.7,3.3 \mathrm{~Hz}, 1 \mathrm{H}), 4.88$ (d, J= 14.6 Hz, $2 \mathrm{H}), 4.74$ (dd, $J=47.0,3.3 \mathrm{~Hz}, 1 \mathrm{H}$ ).

${ }^{13} \mathrm{C}$ NMR $\left(126 \mathrm{MHz}, \mathrm{CDCl}_{3}\right): \delta=164.81,160.05(\mathrm{~d}, J=258.3 \mathrm{~Hz})$, $134.99(\mathrm{q}, J=32.9 \mathrm{~Hz}), 132.86,130.34,125.67(\mathrm{q}, J=3.7 \mathrm{~Hz}), 123.7(\mathrm{q}$, $J=273.4 \mathrm{~Hz}), 95.17(\mathrm{~d}, J=17.2 \mathrm{~Hz}), 62.38(\mathrm{~d}, J=33.8 \mathrm{~Hz})$.

${ }^{19} \mathrm{~F} \mathrm{NMR}\left(376 \mathrm{MHz}, \mathrm{CDCl}_{3}\right): \delta=-63.21,-105.40(\mathrm{dq}, J=47.5,14.9 \mathrm{~Hz})$. HRMS (ESI): $m / z\left[M+\mathrm{H}^{+}\right]$calcd for $\mathrm{C}_{11} \mathrm{H}_{9} \mathrm{~F}_{4} \mathrm{O}_{2}{ }^{+}$: 249.0533; found: 249.0514 .

\section{2-Fluoroallyl Methyl Terephthalate (12a)}

Purified by silica gel chromatography (PE/EtOAc 20:1); colorless oil ${ }^{1} \mathrm{H}$ NMR $\left(400 \mathrm{MHz}, \mathrm{CDCl}_{3}\right): \delta=8.15-8.07(\mathrm{~m}, 4 \mathrm{H}), 4.89$ (dd, $J=15.7$, $3.3 \mathrm{~Hz}, 1 \mathrm{H}), 4.86(\mathrm{~d}, J=14.5 \mathrm{~Hz}, 2 \mathrm{H}), 4.73(\mathrm{dd}, J=47.1,3.3 \mathrm{~Hz}, 1 \mathrm{H})$, $3.95(\mathrm{~s}, 3 \mathrm{H})$.

${ }^{13} \mathrm{C}$ NMR $\left(126 \mathrm{MHz}, \mathrm{CDCl}_{3}\right): \delta=166.33,165.20,160.10(\mathrm{~d}, J=258.2$ $\mathrm{Hz}), 134.41,133.34,129.90,129.77,95.11$ (d, $J=17.2 \mathrm{~Hz}), 62.27$ (d, $J=$ $33.8 \mathrm{~Hz}), 52.63$.

${ }^{19} \mathrm{~F} \mathrm{NMR} \mathrm{(376} \mathrm{MHz,} \mathrm{CDCl}_{3}$ ): $\delta=-102.76$ to $-113.92(\mathrm{~m})$.

HRMS (ESI): $m / z\left[\mathrm{M}+\mathrm{H}^{+}\right]$calcd for $\mathrm{C}_{12} \mathrm{H}_{12} \mathrm{FO}_{4}{ }^{+}$: 239.0714; found: 239.0716 .

\section{2-Fluoroallyl 4-Cyanobenzoate (13a)}

Purified by silica gel chromatography (PE/EtOAc 20:1); colorless oil.

${ }^{1} \mathrm{H} \mathrm{NMR}\left(400 \mathrm{MHz}, \mathrm{CDCl}_{3}\right): \delta=8.16(\mathrm{~d}, J=8.5 \mathrm{~Hz}, 2 \mathrm{H}), 7.76(\mathrm{~d}, J=8.2$ $\mathrm{Hz}, 2 \mathrm{H}), 4.94-4.87(\mathrm{~m}, 1 \mathrm{H}), 4.87$ (d, $J=14.9 \mathrm{~Hz}, 2 \mathrm{H}), 4.73$ (dd, $J=$ 46.9, $3.3 \mathrm{~Hz}, 1 \mathrm{H})$.

${ }^{13} \mathrm{C}$ NMR $\left(126 \mathrm{MHz}, \mathrm{CDCl}_{3}\right): \delta=164.35,159.75(\mathrm{~d}, J=258.3 \mathrm{~Hz})$, 133.36, 132.42, 130.39, 117.96, 116.89, 95.50 (d, $J=16.9 \mathrm{~Hz}), 62.58$ (d, $J=33.1 \mathrm{~Hz})$.

${ }^{19} \mathrm{~F}$ NMR (376 MHz, $\left.\mathrm{CDCl}_{3}\right): \delta=-105.37$.

HRMS (ESI): $m / z\left[\mathrm{M}+\mathrm{H}^{+}\right]$calcd for $\mathrm{C}_{11} \mathrm{H}_{9} \mathrm{FNO}_{2}{ }^{+}$: 206.0612; found: 206.0611.

\section{2-Fluoroallyl 3,5-Bis(trifluoromethyl)benzoate (14a)}

Purified by silica gel chromatography (PE/EtOAc 30:1); colorless oil. ${ }^{1} \mathrm{H} \mathrm{NMR}\left(500 \mathrm{MHz}, \mathrm{CDCl}_{3}\right): \delta=8.51(\mathrm{~s}, 2 \mathrm{H}), 8.09(\mathrm{~s}, 1 \mathrm{H}), 4.94(\mathrm{dd}, J=$ 15.4, 3.4 Hz, $1 \mathrm{H}$ ), 4.92 (d, $J=15.3 \mathrm{~Hz}, 2 \mathrm{H}), 4.77$ (dd, $J=46.7,3.4 \mathrm{~Hz}, 1$ $\mathrm{H})$.

${ }^{13} \mathrm{C}$ NMR (126 MHz, $\left.\mathrm{CDCl}_{3}\right): \delta=163.45,159.56(\mathrm{~d}, J=258.6 \mathrm{~Hz})$, 132.51 (q, $J=34.1 \mathrm{~Hz}), 131.82,130.07(\mathrm{~d}, J=3.2 \mathrm{~Hz}), 126.89$ (pent, $J=$ $3.6 \mathrm{~Hz}), 122.95(\mathrm{q}, J=272.9 \mathrm{~Hz}), 95.96(\mathrm{~d}, J=17.3 \mathrm{~Hz}), 62.98(\mathrm{~d}, J=$ $32.5 \mathrm{~Hz})$.

${ }^{19} \mathrm{~F}$ NMR $\left(471 \mathrm{MHz}, \mathrm{CDCl}_{3}\right): \delta=-63.04,-105.35(\mathrm{dq}, J=48.7,17.2,16.6$ $\mathrm{Hz})$.

HRMS (EI): $m / z$ [M+] calcd for $\mathrm{C}_{12} \mathrm{H}_{7} \mathrm{~F}_{7} \mathrm{O}_{2}{ }^{+}:$316.0334; found: 316.0330 .

\section{2-Fluoroallyl 2-Methoxybenzoate (15a)}

Purified by silica gel chromatography (PE/EtOAc 30:1); colorless oil. ${ }^{1} \mathrm{H}$ NMR $\left(500 \mathrm{MHz}, \mathrm{CDCl}_{3}\right): \delta=7.84(\mathrm{dd}, J=7.9,1.7 \mathrm{~Hz}, 1 \mathrm{H}), 7.52-7.42$ $(\mathrm{m}, 1 \mathrm{H}), 6.98(\mathrm{dt}, J=7.2,3.0 \mathrm{~Hz}, 2 \mathrm{H}), 4.87-4.78(\mathrm{~m}, 3 \mathrm{H}), 4.72(\mathrm{dd}, J=$ 47.6, $3.1 \mathrm{~Hz}, 1 \mathrm{H}), 3.90$ (s, $3 \mathrm{H})$.
${ }^{3} \mathrm{C}$ NMR $\left(126 \mathrm{MHz}, \mathrm{CDCl}_{3}\right): \delta=165.25,160.59(\mathrm{~d}, J=257.4 \mathrm{~Hz}), 159.63$, 134.14, 131.99, 120.27, 119.19, 112.19, 94.12 (d, $J=16.7 \mathrm{~Hz}), 61.50$ (d, $J=35.0 \mathrm{~Hz}), 56.07$.

${ }^{19} \mathrm{~F}$ NMR $\left(471 \mathrm{MHz}, \mathrm{CDCl}_{3}\right): \delta=-94.99$ to $-129.78(\mathrm{~m})$. HRMS (ESI): $m / z\left[\mathrm{M}+\mathrm{H}^{+}\right]$calcd for $\mathrm{C}_{11} \mathrm{H}_{12} \mathrm{FO}_{3}{ }^{+}:$211.0765; found: 211.0771 .

\section{2-Fluoroallyl 2-(11-0xo-6,11-dihydrodibenzo[b,e]oxepin-2-yl)ace- tate (16a)}

Purified by silica gel chromatography (PE/EtOAc 5:1); colorless oil.

${ }^{1} \mathrm{H} \mathrm{NMR}\left(400 \mathrm{MHz}, \mathrm{CDCl}_{3}\right): \delta=8.10(\mathrm{~d}, J=2.1 \mathrm{~Hz}, 1 \mathrm{H}), 7.85(\mathrm{~d}, J=7.6$ $\mathrm{Hz}, 1 \mathrm{H}), 7.51(\mathrm{t}, J=7.1 \mathrm{~Hz}, 1 \mathrm{H}), 7.45-7.37(\mathrm{~m}, 2 \mathrm{H}), 7.31(\mathrm{~d}, J=7.4 \mathrm{~Hz}$, $1 \mathrm{H}), 7.00(\mathrm{~d}, J=8.4 \mathrm{~Hz}, 1 \mathrm{H}), 5.13(\mathrm{~s}, 2 \mathrm{H}), 4.78(\mathrm{dd}, J=15.9,3.3 \mathrm{~Hz}, 1$ H), $4.61(\mathrm{~d}, J=14.3 \mathrm{~Hz}, 2 \mathrm{H}), 4.58(\mathrm{dd}, J=47.4,3.2 \mathrm{~Hz}, 1 \mathrm{H}), 3.68(\mathrm{~s}, 2$ $\mathrm{H})$.

${ }^{13} \mathrm{C}$ NMR $\left(101 \mathrm{MHz}, \mathrm{CDCl}_{3}\right): \delta=190.68,170.65,160.52,159.98(\mathrm{~d}, J=$ $257.8 \mathrm{~Hz}$ ), 140.35, 136.28, 135.49, 132.78, 132.49, 129.42, 129.22, $127.82,127.23,125.10,121.13,94.59(\mathrm{~d}, J=17.0 \mathrm{~Hz}), 73.54,61.65$ (d, $J=33.8 \mathrm{~Hz}), 39.79$.

${ }^{19} \mathrm{~F}$ NMR (376 MHz, $\mathrm{CDCl}_{3}$ ): $\delta=-105.39$ (dq, $J=47.3,14.4 \mathrm{~Hz}$ ).

HRMS (EI): $m / z\left[M+\mathrm{H}^{+}\right]$calcd for $\mathrm{C}_{19} \mathrm{H}_{16} \mathrm{FO}_{4}{ }^{+}$: 327.1027; found: 327.1040 .

\section{2-Fluoroallyl 2-(2-Fluoro-[1,1'-biphenyl]-4-yl)propanoate (17a)} Purified by silica gel chromatography (PE/EtOAc 10:1); colorless oil. ${ }^{1} \mathrm{H} \mathrm{NMR}\left(500 \mathrm{MHz}, \mathrm{CDCl}_{3}\right): \delta=7.58(\mathrm{~d}, J=7.8 \mathrm{~Hz}, 2 \mathrm{H}), 7.49-7.35(\mathrm{~m}, 4$ H), 7.22-7.14 (m, $2 \mathrm{H}), 4.81$ (dd, $J=15.9,3.3 \mathrm{~Hz}, 1 \mathrm{H}), 4.74-4.45(\mathrm{~m}, 3$ $\mathrm{H}), 3.85(\mathrm{q}, J=7.2 \mathrm{~Hz}, 1 \mathrm{H}), 1.60(\mathrm{~d}, J=7.2 \mathrm{~Hz}, 3 \mathrm{H})$.

${ }^{13} \mathrm{C}$ NMR $\left(126 \mathrm{MHz}, \mathrm{CDCl}_{3}\right): \delta=173.30,160.11(\mathrm{~d}, J=258.0 \mathrm{~Hz})$, $159.78(\mathrm{~d}, J=248.4 \mathrm{~Hz}), 141.34(\mathrm{~d}, J=7.5 \mathrm{~Hz}), 135.50,130.96(\mathrm{~d}, J=3.8$ $\mathrm{Hz}), 129.03$ (d, $J=2.7 \mathrm{~Hz}), 128.55,128.08$ (d, $J=13.6 \mathrm{~Hz}), 127.80$, $123.67(\mathrm{~d}, J=3.4 \mathrm{~Hz}), 115.37(\mathrm{~d}, J=23.8 \mathrm{~Hz}), 94.44(\mathrm{~d}, J=16.9 \mathrm{~Hz})$, $61.70(\mathrm{~d}, J=34.2 \mathrm{~Hz}), 44.90,18.40$.

${ }^{19} \mathrm{~F}$ NMR $\left(471 \mathrm{MHz}, \mathrm{CDCl}_{3}\right): \delta=-105.48(\mathrm{dq}, J=47.9,15.1,14.6 \mathrm{~Hz})$, -113.24 to $-132.27(\mathrm{~m})$.

HRMS (EI): $m / z\left[M+\mathrm{H}^{+}\right]$calcd for $\mathrm{C}_{18} \mathrm{H}_{17} \mathrm{~F}_{2} \mathrm{O}_{2}{ }^{+}: 303.1191$; found: 303.1205.

\section{2-Fluoroallyl 2-Bromobenzoate (18a)}

Purified by silica gel chromatography (PE/EtOAc 30:1); colorless oil. ${ }^{1} \mathrm{H} \mathrm{NMR}\left(400 \mathrm{MHz}, \mathrm{CDCl}_{3}\right): \delta=7.83(\mathrm{dd}, J=7.3,2.1 \mathrm{~Hz}, 1 \mathrm{H}), 7.65(\mathrm{dd}$, $J=7.6,1.5 \mathrm{~Hz}, 1 \mathrm{H}), 7.42-7.29$ (m, $2 \mathrm{H}), 4.93-4.66$ ( $\mathrm{m}, 4 \mathrm{H})$.

${ }^{13} \mathrm{C}$ NMR $\left(126 \mathrm{MHz}, \mathrm{CDCl}_{3}\right): \delta=165.26,159.96(\mathrm{~d}, J=258.0 \mathrm{~Hz})$, 134.57, 133.04, 131.65, 131.29, 127.30, 122.04, $95.06(\mathrm{~d}, J=16.8 \mathrm{~Hz})$, $62.26(\mathrm{~d}, J=33.7 \mathrm{~Hz})$.

${ }^{19} \mathrm{~F} \operatorname{NMR}\left(376 \mathrm{MHz}, \mathrm{CDCl}_{3}\right): \delta=-105.19(\mathrm{dq}, J=47.0,14.7 \mathrm{~Hz}$ ).

HRMS (ESI): $m / z\left[\mathrm{M}+\mathrm{H}^{+}\right]$calcd for $\mathrm{C}_{10} \mathrm{H}_{9} \mathrm{BrFO}_{2}: 258.9764$; found: 258.9771

\section{2-Fluoroallyl 5-(2,5-Dimethylphenoxy)-2,2-dimethylpentanoate (19a)}

Purified by silica gel chromatography (PE/EtOAc 30:1); colorless oil. ${ }^{1} \mathrm{H}$ NMR $\left(400 \mathrm{MHz}, \mathrm{CDCl}_{3}\right): \delta=7.06(\mathrm{~d}, J=7.5 \mathrm{~Hz}, 1 \mathrm{H}), 6.72(\mathrm{~d}, J=7.5$ $\mathrm{Hz}, 1 \mathrm{H}), 6.67$ (s, $1 \mathrm{H}), 4.85$ (dd, $J=15.9,3.2 \mathrm{~Hz}, 1 \mathrm{H}), 4.66$ (dd, $J=47.4$, $3.2 \mathrm{~Hz}, 1 \mathrm{H}), 4.66$ (d, J = 13.8 Hz, $2 \mathrm{H}), 4.03-3.86(\mathrm{~m}, 2 \mathrm{H}), 2.37(\mathrm{~s}, 3 \mathrm{H})$, 2.25 (s, $3 \mathrm{H}), 1.96-1.68$ (m, $4 \mathrm{H}), 1.32(\mathrm{~s}, 6 \mathrm{H})$. 
${ }^{13} \mathrm{C}$ NMR $\left(126 \mathrm{MHz}, \mathrm{CDCl}_{3}\right): \delta=177.07,160.61(\mathrm{~d}, J=258.2 \mathrm{~Hz})$, $157.03,136.52,130.39,123.68,120.80,112.02,94.02(d, J=17.1 \mathrm{~Hz})$, 67.90, 61.23 (d, $J=34.0 \mathrm{~Hz}), 42.28,37.16,25.22,25.17,21.48,15.83$.

${ }^{19} \mathrm{~F}$ NMR (376 MHz, $\mathrm{CDCl}_{3}$ ): $\delta=-105.24$ to $-105.65(\mathrm{~m})$.

HRMS (ESI): $m / z\left[\mathrm{M}+\mathrm{H}^{+}\right]$calcd for $\mathrm{C}_{18} \mathrm{H}_{26} \mathrm{FO}_{3}{ }^{+}$: 309.1860; found: 309.1864.

\section{2-Fluoroallyl 2-\{4-[(2-0xocyclopentyl)methyl]phenyl\}propa- noate (20a)}

Purified by silica gel chromatography (PE/EtOAc 10:1), colorless oil; $\mathrm{dr}=1: 1$.

${ }^{1} \mathrm{H}$ NMR $\left(500 \mathrm{MHz}, \mathrm{CDCl}_{3}\right): \delta=7.20(\mathrm{~d}, J=8.1 \mathrm{~Hz}, 2 \mathrm{H}), 7.10(\mathrm{~d}, J=8.1$ $\mathrm{Hz}, 2 \mathrm{H}$ ), 4.70 (dd, $J=16.1,3.3 \mathrm{~Hz}, 1 \mathrm{H}), 4.61-4.36(\mathrm{~m}, 3 \mathrm{H}), 3.73(\mathrm{q}, J=$ $7.2 \mathrm{~Hz}, 1 \mathrm{H}), 3.10(\mathrm{dd}, J=13.9,4.1 \mathrm{~Hz}, 1 \mathrm{H}), 2.49(\mathrm{dd}, J=13.9,9.5 \mathrm{~Hz}, 1$ H), 2.37-2.27 (m, $2 \mathrm{H}), 2.13-2.00(\mathrm{~m}, 2 \mathrm{H}), 1.97-1.87(\mathrm{~m}, 1 \mathrm{H}), 1.70$ (dtdd, $J=12.8,10.6,8.4,6.4 \mathrm{~Hz}, 1 \mathrm{H}), 1.59-1.40(\mathrm{~m}, 1 \mathrm{H}), 1.48$ (d, $J=$ $7.2 \mathrm{~Hz}, 3 \mathrm{H})$.

${ }^{13} \mathrm{C}$ NMR $\left(126 \mathrm{MHz}, \mathrm{CDCl}_{3}\right): \delta=220.31,173.84,160.20(\mathrm{~d}, J=257.5$ $\mathrm{Hz}), 139.07,137.83,129.20,127.56,93.89$ (d, $J=17.0 \mathrm{~Hz}), 61.29$ (d, $J=$ $34.9 \mathrm{~Hz}$ ), 50.95, 44.93, 38.18, 35.18, 29.17, 20.55, 18.39 (d, $J=1.3 \mathrm{~Hz}$ ). $\left.{ }^{19} \mathrm{~F} \mathrm{NMR} \mathrm{(471} \mathrm{MHz,} \mathrm{CDCl}_{3}\right): \delta=-88.67$ to $-129.99(\mathrm{~m})$.

HRMS (ESI): $m / z\left[\mathrm{M}+\mathrm{H}^{+}\right]$calcd for $\mathrm{C}_{18} \mathrm{H}_{22} \mathrm{FO}_{3}{ }^{+}$: 305.1547; found: 305.1556.

\section{Cobalt-Catalyzed Hydrocyanation of Monofluoroalkenes; General Procedure (GP)}

A $25 \mathrm{~mL}$ oven-dried Schlenk tube equipped with a magnetic stirring bar was charged with the respective monofluoroalkene $(0.1 \mathrm{mmol}$ or $0.5 \mathrm{mmol})$, tosyl cyanide $(0.3 \mathrm{mmol}$ or $1.5 \mathrm{mmol})$, and $\mathrm{Co}^{\mathrm{II}} \mathrm{Sal}^{\mathrm{t}-\mathrm{Bu}, \mathrm{t}-\mathrm{Bu}}$ (0.01 mmol or $0.05 \mathrm{mmol})$, EtOH ( $1 \mathrm{~mL}$ or $5 \mathrm{~mL}$ ). Then the $\mathrm{PhSiH}_{3}$ $(0.25 \mathrm{mmol}$ or $1.25 \mathrm{mmol})$ and TBHP $(0.03 \mathrm{mmol}$ or $0.15 \mathrm{mmol})$ were added dropwise in sequence. The reaction mixture was stirred at r.t. for $12 \mathrm{~h}$. Then the mixture was concentrated directly in vacuo for purification by column chromatography on silica gel (eluent: PE/EtOAc) to provide the respective products $\mathbf{2 - 2 0}$.

\section{3-(1,3-Dioxoisoindolin-2-yl)-2-fluoro-2-methylpropanenitrile (2)}

Prepared by following GP using $\mathbf{1}(20.5 \mathrm{mg}, 0.1 \mathrm{mmol})$ as substrate. Purification by flash column chromatography (silica gel, PE:EtOAc 10:1); white solid; yield: $19.0 \mathrm{mg}(82 \%)$; mp $120.0-123.3^{\circ} \mathrm{C}$.

${ }^{1} \mathrm{H} \mathrm{NMR}\left(400 \mathrm{MHz}, \mathrm{CDCl}_{3}\right): \delta=7.92$ (dd, $\left.J=5.5,3.1 \mathrm{~Hz}, 2 \mathrm{H}\right), 7.79$ (dd, $J=5.5,3.1 \mathrm{~Hz}, 2 \mathrm{H}), 4.29-4.08$ (m, $2 \mathrm{H}), 1.83$ (d, $J=21.2 \mathrm{~Hz}, 3 \mathrm{H})$.

${ }^{13} \mathrm{C}$ NMR $\left(126 \mathrm{MHz}, \mathrm{CDCl}_{3}\right): \delta=167.44,134.76,131.67,124.08$, $116.70(\mathrm{~d}, J=33.6 \mathrm{~Hz}), 86.97(\mathrm{~d}, J=186.7 \mathrm{~Hz}), 44.13(\mathrm{~d}, J=26.2 \mathrm{~Hz})$, $23.93(\mathrm{~d}, J=24.0 \mathrm{~Hz})$.

${ }^{19} \mathrm{~F}$ NMR (376 MHz, $\mathrm{CDCl}_{3}$ ): $\delta=-145.09$ to $-155.55(\mathrm{~m})$.

HRMS (ESI): $m / z\left[\mathrm{M}+\mathrm{H}^{+}\right]$calcd for $\mathrm{C}_{12} \mathrm{H}_{10} \mathrm{FN}_{2} \mathrm{O}_{2}{ }^{+}:$233.0721; found: 233.0731 .

\section{2-Fluoro-3-mesityl-2-methylpropanenitrile (3)}

Prepared by following GP using $3 \mathbf{a}$ ( $89 \mathrm{mg}, 0.5 \mathrm{mmol}$ ) as substrate. Purification by silica gel chromatography (PE); colorless oil; yield: 63.5 $\mathrm{mg}(62 \%)$.

${ }^{1} \mathrm{H}$ NMR $\left(500 \mathrm{MHz}, \mathrm{CDCl}_{3}\right): \delta=6.94(\mathrm{~s}, 2 \mathrm{H}), 3.54-3.17(\mathrm{~m}, 2 \mathrm{H}), 2.39$ (s, $6 \mathrm{H}), 2.31(\mathrm{~s}, 3 \mathrm{H}), 1.83(\mathrm{~d}, J=21.2 \mathrm{~Hz}, 3 \mathrm{H})$.
${ }^{13} \mathrm{C}$ NMR $\left(126 \mathrm{MHz}, \mathrm{CDCl}_{3}\right): \delta=138.13,137.30,129.59,127.11,118.66$ $(\mathrm{d}, J=35.0 \mathrm{~Hz}), 89.27$ (d, $J=182.1 \mathrm{~Hz}), 38.47$ (d, $J=23.5 \mathrm{~Hz}), 26.14$ (d, $J=25.4 \mathrm{~Hz}), 20.92,20.90$.

${ }^{19} \mathrm{~F} \mathrm{NMR}\left(471 \mathrm{MHz}, \mathrm{CDCl}_{3}\right): \delta=-134.04$ to $-145.79(\mathrm{~m})$.

HRMS (EI): $m / z$ [ $\left[\mathrm{M}^{+}\right]$calcd for $\mathrm{C}_{13} \mathrm{H}_{16} \mathrm{FN}^{+}:$205.1267; found: 205.1261 .

\section{Methyl 4-[(4-Cyano-4-fluoropentyl)oxy]benzoate (4)}

Prepared by following GP using $\mathbf{4 a}(23.8 \mathrm{mg}, 0.1 \mathrm{mmol})$ as substrate. Purification by flash column chromatography (silica gel, PE:EtOAc 10:1); colorless oil; yield: $20.2 \mathrm{mg}(76 \%)$.

${ }^{1} \mathrm{H}$ NMR $\left(500 \mathrm{MHz}, \mathrm{CDCl}_{3}\right): \delta=7.99(\mathrm{~d}, J=8.8 \mathrm{~Hz}, 2 \mathrm{H}), 6.90(\mathrm{~d}, J=8.9$ $\mathrm{Hz}, 2 \mathrm{H}), 4.09$ (qt, J = 9.7, $5.2 \mathrm{~Hz}, 2 \mathrm{H}$ ), 3.89 (s, $3 \mathrm{H}), 2.38-1.99$ (m, $4 \mathrm{H}$ ), $1.80(\mathrm{~d}, J=21.1 \mathrm{~Hz}, 3 \mathrm{H})$.

${ }^{13} \mathrm{C}$ NMR $\left(126 \mathrm{MHz}, \mathrm{CDCl}_{3}\right): \delta=166.94,162.49,131.79,123.01,118.14$ (d, $J=34.8 \mathrm{~Hz}), 114.13,88.31$ (d, $J=180.2 \mathrm{~Hz}), 66.81,52.06,36.84$ (d, $J=23.1 \mathrm{~Hz}), 25.94(\mathrm{~d}, J=24.8 \mathrm{~Hz}), 24.02(\mathrm{~d}, J=3.7 \mathrm{~Hz})$.

${ }^{19} \mathrm{~F}$ NMR (471 MHz, $\mathrm{CDCl}_{3}$ ): $\delta=-146.15$ (dddd, $J=27.3,20.9,13.4,5.8$ $\mathrm{Hz})$.

HRMS (ESI): $m / z\left[\mathrm{M}+\mathrm{H}^{+}\right]$calcd for $\mathrm{C}_{14} \mathrm{H}_{17} \mathrm{FNO}_{3}{ }^{+}:$266.1187; found: 266.1195.

\section{$\mathrm{N}$-(2-Cyano-2-fluoropropyl)- $\mathrm{N}$-phenylbenzamide (5)}

Prepared by following GP using $\mathbf{5 a}(25.5 \mathrm{mg}, 0.1 \mathrm{mmol})$ as substrate. Purification by flash column chromatography (silica gel, PE:EtOAc 10:1); colorless oil; yield: $13.0 \mathrm{mg}(46 \%)$.

${ }^{1} \mathrm{H}$ NMR $\left(400 \mathrm{MHz}, \mathrm{CDCl}_{3}\right): \delta=7.33-7.21(\mathrm{~m}, 5 \mathrm{H}), 7.20-7.10(\mathrm{~m}, 5 \mathrm{H})$, 4.66-4.30 (m, $2 \mathrm{H}), 1.86$ (d, $J=21.5 \mathrm{~Hz}, 3 \mathrm{H})$.

${ }^{13} \mathrm{C}$ NMR $\left(126 \mathrm{MHz}, \mathrm{CDCl}_{3}\right): \delta=171.55,143.21,134.92,130.27$, $129.51,128.83,127.98,127.55,117.12(\mathrm{~d}, J=34.2 \mathrm{~Hz}), 88.65(\mathrm{~d}, J=$ $183.7 \mathrm{~Hz}), 55.68(\mathrm{~d}, J=23.8 \mathrm{~Hz}), 29.84,24.07(\mathrm{~d}, J=24.3 \mathrm{~Hz})$.

${ }^{19} \mathrm{~F} \mathrm{NMR}\left(376 \mathrm{MHz}, \mathrm{CDCl}_{3}\right): \delta=-143.61(\mathrm{dq}, J=43.4,21.6 \mathrm{~Hz}$ ). HRMS (ESI): $m / z\left[\mathrm{M}+\mathrm{H}^{+}\right]$calcd for $\mathrm{C}_{17} \mathrm{H}_{16} \mathrm{FN}_{2} \mathrm{O}^{+}$: 283.1241; found: 283.1254 .

\section{2-Cyano-2-fluoropropyl Benzoate (6)}

Prepared by following GP using $\mathbf{6 a}(90 \mathrm{mg}, 0.5 \mathrm{mmol}$ ) as substrate. Purification by flash column chromatography ( silica gel, PE:EtOAc 30:1); colorless oil; yield: $74.5 \mathrm{mg}$ (72\%).

${ }^{1} \mathrm{H}$ NMR $\left(400 \mathrm{MHz}, \mathrm{CDCl}_{3}\right): \delta=8.25-7.81(\mathrm{~m}, 2 \mathrm{H}), 7.55(\mathrm{tt}, J=7.0,1.3$ $\mathrm{Hz}, 1 \mathrm{H}), 7.47-7.34$ (m, $2 \mathrm{H}), 4.73-4.29$ (m, $2 \mathrm{H}), 1.79$ (d, J = $20.9 \mathrm{~Hz}, 3$ $\mathrm{H})$.

${ }^{13} \mathrm{C}$ NMR $\left(101 \mathrm{MHz}, \mathrm{CDCl}_{3}\right): \delta=165.42,133.95,130.06,128.76$, $128.70,116.36(\mathrm{~d}, J=34.4 \mathrm{~Hz}), 86.31(\mathrm{~d}, J=184.7 \mathrm{~Hz}), 66.62(\mathrm{~d}, J=25.7$ $\mathrm{Hz}), 22.59(\mathrm{~d}, J=24.0 \mathrm{~Hz})$.

${ }^{19} \mathrm{~F} \operatorname{NMR}\left(376 \mathrm{MHz}, \mathrm{CDCl}_{3}\right.$ ): $\delta=-152.39(\mathrm{pd}, J=21.3,14.8 \mathrm{~Hz}$ ). HRMS (ESI): $m / z\left[\mathrm{M}+\mathrm{H}^{+}\right]$calcd for $\mathrm{C}_{11} \mathrm{H}_{11} \mathrm{FNO}_{2}{ }^{+}:$208.0768; found: 208.0775 .

\section{2-Cyano-2-fluoropropyl 4-Methylbenzoate (7)}

Prepared by following GP using 7a (97 mg, $0.5 \mathrm{mmol}$ ) as substrate. Purification by flash column chromatography (silica gel, PE:EtOAc 30:1); colorless oil; yield: $86.2 \mathrm{mg}$ (78\%).

${ }^{1} \mathrm{H}$ NMR $\left(500 \mathrm{MHz}, \mathrm{CDCl}_{3}\right): \delta=7.98(\mathrm{~d}, J=8.1 \mathrm{~Hz}, 2 \mathrm{H}), 7.27$ (d, $J=8.1$ $\mathrm{Hz}, 2 \mathrm{H}), 4.88-4.28(\mathrm{~m}, 2 \mathrm{H}), 2.43(\mathrm{~s}, 3 \mathrm{H}), 1.86(\mathrm{~d}, J=20.9 \mathrm{~Hz}, 3 \mathrm{H})$. 
${ }^{13} \mathrm{C}$ NMR $\left(126 \mathrm{MHz}, \mathrm{CDCl}_{3}\right): \delta=165.49,144.85,130.11,129.81$, $129.48,116.42(\mathrm{~d}, J=34.5 \mathrm{~Hz}), 86.36(\mathrm{~d}, J=184.5 \mathrm{~Hz}), 66.47(\mathrm{~d}, J=25.9$ $\mathrm{Hz}$ ), 22.60 (d, $J=24.2 \mathrm{~Hz}$ ), 21.89 .

${ }^{19} \mathrm{~F}$ NMR (376 MHz, $\left.\mathrm{CDCl}_{3}\right): \delta=-152.39(\mathrm{pd}, J=21.0,15.0 \mathrm{~Hz}$ ).

HRMS (ESI): $m / z\left[\mathrm{M}+\mathrm{H}^{+}\right]$calcd for $\mathrm{C}_{12} \mathrm{H}_{13} \mathrm{FNO}_{2}{ }^{+}$: 222.0925; found: 222.0938 .

\section{2-Cyano-2-fluoropropyl 4-Isopropylbenzoate (8)}

Prepared by following GP using $\mathbf{8 a}(111 \mathrm{mg}, 0.5 \mathrm{mmol})$ as substrate. Purification by flash column chromatography (silica gel, PE:EtOAc 30:1); colorless oil; yield: $101.0 \mathrm{mg}(81 \%)$.

${ }^{1} \mathrm{H}$ NMR $\left(500 \mathrm{MHz}, \mathrm{CDCl}_{3}\right): \delta=8.02(\mathrm{~d}, J=8.2 \mathrm{~Hz}, 2 \mathrm{H}), 7.33(\mathrm{~d}, J=8.2$ $\mathrm{Hz}, 2 \mathrm{H}$ ), 4.81-4.34 (m, $2 \mathrm{H}), 2.97$ (dq, $J=13.7,6.9 \mathrm{~Hz}, 1 \mathrm{H}), 1.86$ (d, $J=$ $20.9 \mathrm{~Hz}, 3 \mathrm{H}), 1.27$ (d, $J=6.9 \mathrm{~Hz}, 6 \mathrm{H})$.

${ }^{13} \mathrm{C}$ NMR $\left(126 \mathrm{MHz}, \mathrm{CDCl}_{3}\right): \delta=165.48,155.58,130.29,126.90$, $126.28,116.42$ (d, $J=34.4 \mathrm{~Hz}), 86.37$ (d, $J=184.8 \mathrm{~Hz}), 66.45$ (d, $J=25.8$ $\mathrm{Hz}), 34.47,23.80,22.61(\mathrm{~d}, J=24.0 \mathrm{~Hz})$.

${ }^{19} \mathrm{~F}$ NMR (471 MHz, $\left.\mathrm{CDCl}_{3}\right): \delta=-152.41(\mathrm{pd}, J=20.9,15.3 \mathrm{~Hz}$ ).

HRMS (ESI): $m / z\left[\mathrm{M}+\mathrm{H}^{+}\right]$calcd for $\mathrm{C}_{14} \mathrm{H}_{17} \mathrm{FNO}_{2}{ }^{+}:$250.1238; found: 250.1245 .

\section{2-Cyano-2-fluoropropyl 4-Methoxybenzoate (9)}

Prepared by following GP using 9a (105 $\mathrm{mg}, 0.5 \mathrm{mmol}$ ) as substrate. Purification by flash column chromatography (silica gel, PE:EtOAc 20:1); colorless oil; yield: $71.1 \mathrm{mg}(60 \%)$.

${ }^{1} \mathrm{H} \mathrm{NMR}\left(400 \mathrm{MHz}, \mathrm{CDCl}_{3}\right): \delta=8.25-7.93(\mathrm{~m}, 2 \mathrm{H}), 7.05-6.68(\mathrm{~m}, 2 \mathrm{H})$, 4.83-4.14 (m, $2 \mathrm{H}), 3.88$ (s, $3 \mathrm{H}), 1.85(\mathrm{~d}, J=20.9 \mathrm{~Hz}, 3 \mathrm{H})$.

${ }^{13} \mathrm{C} \mathrm{NMR}\left(126 \mathrm{MHz}, \mathrm{CDCl}_{3}\right): \delta=165.14,164.18,132.22,121.02,116.46$ $(\mathrm{d}, J=34.2 \mathrm{~Hz}), 114.04,86.42(\mathrm{~d}, J=184.6 \mathrm{~Hz}), 66.39(\mathrm{~d}, J=25.9 \mathrm{~Hz})$, $55.65,22.62(\mathrm{~d}, J=24.0 \mathrm{~Hz})$.

${ }^{19} \mathrm{~F} \mathrm{NMR}\left(471 \mathrm{MHz}, \mathrm{CDCl}_{3}\right): \delta=-152.35(\mathrm{dtd}, J=41.8,20.8,11.3 \mathrm{~Hz})$. HRMS (ESI): $m / z\left[\mathrm{M}+\mathrm{H}^{+}\right]$calcd for $\mathrm{C}_{12} \mathrm{H}_{13} \mathrm{FNO}_{3}{ }^{+}:$238.0874; found: 238.0884 .

\section{2-Cyano-2-fluoropropyl [1,1'-Biphenyl]-4-carboxylate (10)}

Prepared by following GP using $\mathbf{1 0 a}(25.6 \mathrm{mg}, 0.1 \mathrm{mmol})$ as substrate. Purification by flash column chromatography (silica gel, PE:EtOAc 30:1); white solid; yield: $17.8 \mathrm{mg}(63 \%)$; $\mathrm{mp} 105.9-107.2^{\circ} \mathrm{C}$.

${ }^{1} \mathrm{H} \mathrm{NMR}\left(500 \mathrm{MHz}, \mathrm{CDCl}_{3}\right): \delta=8.22-8.09(\mathrm{~m}, 2 \mathrm{H}), 7.72-7.67(\mathrm{~m}, 2 \mathrm{H})$, 7.65-7.59 (m, $2 \mathrm{H}), 7.49(\mathrm{t}, J=7.5 \mathrm{~Hz}, 2 \mathrm{H}), 7.42(\mathrm{t}, J=7.3,6.4,3.2 \mathrm{~Hz}, 1$ H), 4.75-4.49 (m, $2 \mathrm{H}), 1.88$ (d, $J=20.9 \mathrm{~Hz}, 3 \mathrm{H})$.

${ }^{13} \mathrm{C}$ NMR $\left(126 \mathrm{MHz}, \mathrm{CDCl}_{3}\right): \delta=165.34,146.72,139.87,130.62$, $129.13,128.51,127.45,127.43,127.39,116.40(\mathrm{~d}, J=34.6 \mathrm{~Hz}), 86.36$ (d, $J=184.8 \mathrm{~Hz}), 66.64(\mathrm{~d}, J=25.7 \mathrm{~Hz}), 22.62(\mathrm{~d}, J=24.0 \mathrm{~Hz})$.

$\left.{ }^{19} \mathrm{~F} \mathrm{NMR} \mathrm{(471} \mathrm{MHz,} \mathrm{CDCl}_{3}\right): \delta=-152.30(\mathrm{pd}, J=21.5,15.0 \mathrm{~Hz})$. HRMS (ESI): $m / z$ [M + Na+] calcd for $\mathrm{C}_{17} \mathrm{H}_{14} \mathrm{FNO}_{2} \mathrm{Na}^{+}: 306.0901$; found: 306.0905.

\section{2-Cyano-2-fluoropropyl 4-(Trifluoromethyl)benzoate (11)}

Prepared by following GP using 11a (124 mg, $0.5 \mathrm{mmol}$ ) as substrate. Purification by flash column chromatography (silica gel, PE:EtOAc 30:1); colorless oil; yield: $68.8 \mathrm{mg}(50 \%)$.

${ }^{1} \mathrm{H}$ NMR $\left(500 \mathrm{MHz}, \mathrm{CDCl}_{3}\right): \delta=8.21(\mathrm{~d}, J=8.1 \mathrm{~Hz}, 2 \mathrm{H}), 7.75(\mathrm{~d}, J=8.2$ $\mathrm{Hz}, 2 \mathrm{H}), 4.90-4.12$ (m, $2 \mathrm{H}), 1.88$ (d, $J=20.8 \mathrm{~Hz}, 3 \mathrm{H})$.
${ }^{13} \mathrm{C}$ NMR $\left(126 \mathrm{MHz}, \mathrm{CDCl}_{3}\right): \delta=164.28,135.38(\mathrm{q}, J=32.8 \mathrm{~Hz}), 131.93$, 130.50, $125.84(\mathrm{q}, J=3.7 \mathrm{~Hz}), 123.59(\mathrm{q}, J=273.0 \mathrm{~Hz}), 116.18(\mathrm{~d}, J=$ $34.4 \mathrm{~Hz}), 86.21$ (d, $J=185.4 \mathrm{~Hz}), 67.05$ (d, $J=25.5 \mathrm{~Hz}), 22.52$ (d, $J=$ $23.9 \mathrm{~Hz})$.

${ }^{19} \mathrm{~F}$ NMR (471 MHz, $\mathrm{CDCl}_{3}$ ): $\delta=-63.24,-152.35(\mathrm{pd}, J=21.1,14.3 \mathrm{~Hz}$ ). HRMS (ESI): $m / z$ [M + Na+] calcd for $\mathrm{C}_{12} \mathrm{H}_{9} \mathrm{~F}_{4} \mathrm{NO}_{2} \mathrm{Na}^{+}: 298.0462$; found: 298.0459 .

\section{2-Cyano-2-fluoropropyl Methyl Terephthalate (12)}

Prepared by following GP using 12a (119 $\mathrm{mg}, 0.5 \mathrm{mmol})$ as substrate. Purification by flash column chromatography (silica gel, PE:EtOAc 20:1); colorless oil; yield: $79.5 \mathrm{mg}(60 \%)$.

${ }^{1} \mathrm{H} \mathrm{NMR}\left(400 \mathrm{MHz}, \mathrm{CDCl}_{3}\right): \delta=8.28-7.99(\mathrm{~m}, 4 \mathrm{H}), 4.80-4.37(\mathrm{~m}, 2 \mathrm{H})$, $3.96(\mathrm{~s}, 3 \mathrm{H}), 1.88(\mathrm{~d}, J=20.9 \mathrm{~Hz}, 3 \mathrm{H})$.

${ }^{13} \mathrm{C}$ NMR $\left(101 \mathrm{MHz}, \mathrm{CDCl}_{3}\right): \delta=166.18,164.66,134.82,132.40$, 130.04, 129.88, 116.23 (d, $J=34.5 \mathrm{~Hz}), 86.22$ (d, $J=185.0 \mathrm{~Hz}), 66.95$ $(\mathrm{d}, J=25.5 \mathrm{~Hz}), 52.68,22.56(\mathrm{~d}, J=24.0 \mathrm{~Hz})$.

${ }^{19} \mathrm{~F}$ NMR (376 MHz, $\mathrm{CDCl}_{3}$ ): $\delta=-152.35$ (pd, $J=20.9,14.6 \mathrm{~Hz}$ ). HRMS (ESI): $m / z\left[\mathrm{M}+\mathrm{H}^{+}\right]$calcd for $\mathrm{C}_{13} \mathrm{H}_{13} \mathrm{FNO}_{4}{ }^{+}$: 266.0823; found: 266.0833 .

\section{2-Cyano-2-fluoropropyl 4-Cyanobenzoate (13)}

Prepared by following GP using 13a (20.5 mg, $0.1 \mathrm{mmol})$ as substrate. Purification by flash column chromatography (silica gel, PE:EtOAc 20:1); white solid; yield: $14.0 \mathrm{mg}(60 \%) ; \mathrm{mp} 91.0-92.7^{\circ} \mathrm{C}$.

${ }^{1} \mathrm{H} \mathrm{NMR}\left(400 \mathrm{MHz}, \mathrm{CDCl}_{3}\right): \delta=8.54-8.03(\mathrm{~m}, 2 \mathrm{H}), 8.02-7.56(\mathrm{~m}, 2 \mathrm{H})$, $5.22-4.23(\mathrm{~m}, 2 \mathrm{H}), 1.87$ (d, $J=20.8 \mathrm{~Hz}, 3 \mathrm{H}$ ).

${ }^{13} \mathrm{C}$ NMR $\left(126 \mathrm{MHz}, \mathrm{CDCl}_{3}\right): \delta=163.76,132.49,132.38,130.46$, 117.75, 117.29, 115.99 (d, $J=34.7 \mathrm{~Hz}), 86.05$ (d, $J=185.5 \mathrm{~Hz}), 67.13$ (d, $J=24.9 \mathrm{~Hz}), 22.40$ (d, $J=24.0 \mathrm{~Hz})$.

${ }^{19} \mathrm{~F}$ NMR (376 MHz, $\mathrm{CDCl}_{3}$ ): $\delta=-148.37$ to $-156.53(\mathrm{~m})$, HRMS (ESI): $m / z\left[\mathrm{M}+\mathrm{H}^{+}\right]$calcd for $\mathrm{C}_{12} \mathrm{H}_{10} \mathrm{FN}_{2} \mathrm{O}_{2}{ }^{+}$: 233.0721; found: 233.0720 .

2-Cyano-2-fluoropropyl 3,5-Bis(trifluoromethyl)benzoate (14) Prepared by following GP using $\mathbf{1 4 a}(158 \mathrm{mg}, 0.5 \mathrm{mmol}$ ) as substrate. Purification by flash column chromatography (silica gel, PE:EtOAc 30:1); colorless oil; yield: $108.1 \mathrm{mg}(63 \%)$.

${ }^{1} \mathrm{H} \mathrm{NMR}\left(500 \mathrm{MHz}, \mathrm{CDCl}_{3}\right): \delta=8.52(\mathrm{~s}, 2 \mathrm{H}), 8.13(\mathrm{~s}, 1 \mathrm{H}), 4.81-4.47(\mathrm{~m}$, $2 \mathrm{H}), 1.89(\mathrm{~d}, J=20.8 \mathrm{~Hz}, 3 \mathrm{H})$.

${ }^{13} \mathrm{C}$ NMR $\left(126 \mathrm{MHz}, \mathrm{CDCl}_{3}\right): \delta=162.95,132.69(\mathrm{q}, J=34.3 \mathrm{~Hz}), 130.95$, $130.12(\mathrm{~d}, J=3.2 \mathrm{~Hz}), 127.72-126.99(\mathrm{~m}), 122.82(\mathrm{q}, J=273.0 \mathrm{~Hz})$, $115.95(\mathrm{~d}, J=34.4 \mathrm{~Hz}), 86.10(\mathrm{~d}, J=185.6 \mathrm{~Hz}), 67.42(\mathrm{~d}, J=24.9 \mathrm{~Hz})$, $22.44(\mathrm{~d}, J=24.0 \mathrm{~Hz})$.

${ }^{19} \mathrm{~F}$ NMR (471 MHz, $\mathrm{CDCl}_{3}$ ): $\delta=-63.24,-152.35(\mathrm{pd}, J=21.1,14.3 \mathrm{~Hz}$ ). HRMS (ESI): $m / z\left[\mathrm{M}+\mathrm{Na}^{+}\right]$calcd for $\mathrm{C}_{13} \mathrm{H}_{8} \mathrm{~F}_{7} \mathrm{NO}_{2} \mathrm{Na}^{+}: 366.0335$; found: 366.0365 .

\section{2-Cyano-2-fluoropropyl 2-Methoxybenzoate (15)}

Prepared by following GP using 15a (105 mg, $0.5 \mathrm{mmol}$ ) as substrate. Purification by flash column chromatography (silica gel, PE:EtOAc 20:1); colorless oil; yield: $61.6 \mathrm{mg}(52 \%)$.

${ }^{1} \mathrm{H}$ NMR $\left(500 \mathrm{MHz}, \mathrm{CDCl}_{3}\right): \delta=7.90(\mathrm{~d}, J=7.8 \mathrm{~Hz}, 1 \mathrm{H}), 7.53(\mathrm{t}, J=7.8$ $\mathrm{Hz}, 1 \mathrm{H}), 7.01(\mathrm{t}, J=7.8 \mathrm{~Hz}, 2 \mathrm{H}), 4.75-4.38(\mathrm{~m}, 2 \mathrm{H}), 3.93(\mathrm{~s}, 3 \mathrm{H}), 1.86$ $(\mathrm{d}, J=21.0 \mathrm{~Hz}, 3 \mathrm{H})$. 
${ }^{13} \mathrm{C}$ NMR $\left(126 \mathrm{MHz}, \mathrm{CDCl}_{3}\right): \delta=164.86,159.92,134.74,132.29$, 120.39, 118.17, 116.55 (d, $J=34.2 \mathrm{~Hz}), 112.22,86.35$ (d, $J=184.1 \mathrm{~Hz})$, $66.43(\mathrm{~d}, J=26.5 \mathrm{~Hz}), 56.05,22.68(\mathrm{~d}, J=23.9 \mathrm{~Hz})$.

${ }^{19} \mathrm{~F}$ NMR (471 MHz, $\left.\mathrm{CDCl}_{3}\right): \delta=-152.34(\mathrm{pd}, J=20.9,14.7 \mathrm{~Hz})$.

HRMS (ESI): $m / z\left[\mathrm{M}+\mathrm{Na}^{+}\right]$calcd for $\mathrm{C}_{12} \mathrm{H}_{12} \mathrm{FNO}_{3} \mathrm{Na}^{+}: 260.0693$; found: 260.0705 .

\section{2-Cyano-2-fluoropropyl 2-(11-0xo-6,11-dihydrodibenzo[b,e]ox- epin-2-yl)acetate (16)}

Prepared by following GP using $\mathbf{1 6 a}(32.6 \mathrm{mg}, 0.1 \mathrm{mmol})$ as substrate. Purification by flash column chromatography (silica gel, PE:EtOAc 10:1); colorless oil; yield: $14.5 \mathrm{mg}(41 \%)$.

${ }^{1} \mathrm{H}$ NMR $\left(400 \mathrm{MHz}, \mathrm{CDCl}_{3}\right): \delta=8.13(\mathrm{~d}, J=2.3 \mathrm{~Hz}, 1 \mathrm{H}), 7.88(\mathrm{~d}, J=7.6$ $\mathrm{Hz}, 1 \mathrm{H}), 7.56(\mathrm{td}, J=7.4,1.1 \mathrm{~Hz}, 1 \mathrm{H}), 7.50-7.41(\mathrm{~m}, 2 \mathrm{H}), 7.37(\mathrm{~d}, J=$ $7.4 \mathrm{~Hz}, 1 \mathrm{H}), 7.05(\mathrm{~d}, J=8.4 \mathrm{~Hz}, 1 \mathrm{H}), 5.19(\mathrm{~s}, 2 \mathrm{H}), 4.63-4.15(\mathrm{~m}, 2 \mathrm{H})$, $3.76(\mathrm{~s}, 2 \mathrm{H}), 1.76$ (d, $J=20.9 \mathrm{~Hz}, 3 \mathrm{H})$.

${ }^{13} \mathrm{C}$ NMR $\left(126 \mathrm{MHz}, \mathrm{CDCl}_{3}\right): \delta=190.90,170.35,160.81,140.51$, $136.40,135.60,132.97,132.70,129.61,129.43,127.97,126.79$, 125.31, 121.42, 116.19 (d, $J=34.2 \mathrm{~Hz}), 86.09$ (d, $J=184.8 \mathrm{~Hz}), 73.76$, $66.49(\mathrm{~d}, J=25.7 \mathrm{~Hz}), 39.78,22.46(\mathrm{~d}, J=23.9 \mathrm{~Hz})$.

${ }^{19} \mathrm{~F}$ NMR (376 MHz, $\mathrm{CDCl}_{3}$ ): $\delta=-152.60$ (pd, $J=21.3,15.3 \mathrm{~Hz}$ ).

HRMS (ESI): $m / z\left[\mathrm{M}+\mathrm{H}^{+}\right]$calcd for $\mathrm{C}_{20} \mathrm{H}_{17} \mathrm{FNO}_{4}^{+}$: 354.1136; found: 354.1145 .

\section{2-Cyano-2-fluoropropyl 2-(2-Fluoro-[1,1'-biphenyl]-4-yl)propa- noate (17)}

Prepared by following GP using $\mathbf{1 7 a}(30.2 \mathrm{mg}, 0.1 \mathrm{mmol})$ as substrate. Purification by flash column chromatography (silica gel, PE:EtOAc 10:1); colorless oil; yield: $13.5 \mathrm{mg}(41 \%) ; \mathrm{dr}=1: 1$.

${ }^{1} \mathrm{H}$ NMR $\left(400 \mathrm{MHz}, \mathrm{CDCl}_{3}\right): \delta=7.58-7.53(\mathrm{~m}, 2 \mathrm{H}), 7.51-7.42(\mathrm{~m}, 2 \mathrm{H})$, 7.41-7.33 (m, $2 \mathrm{H}), 7.21-7.09$ ( $\mathrm{m}, 2 \mathrm{H}), 4.61-4.15$ ( $\mathrm{m}, 2 \mathrm{H}), 3.88$ (q, $J=$ $7.2 \mathrm{~Hz}, 1 \mathrm{H}), 1.72$ (d, J = 20.8 Hz, $3 \mathrm{H}), 1.61$ (d, $J=7.2 \mathrm{~Hz}, 3 \mathrm{H}$ ).

${ }^{13} \mathrm{C}$ NMR $\left(126 \mathrm{MHz}, \mathrm{CDCl}_{3}\right): \delta=172.94,172.89,159.84(\mathrm{~d}, J=248.8$ $\mathrm{Hz}), 140.75(\mathrm{~d}, J=2.3 \mathrm{~Hz}), 140.69(\mathrm{~d}, J=2.4 \mathrm{~Hz}), 135.46,131.11$, 131.08, 129.09, 129.07, 128.60, 128.35 (d, $J=13.5 \mathrm{~Hz}), 127.89,123.78$ $(\mathrm{t}, J=3.6 \mathrm{~Hz}), 116.15(\mathrm{dd}, J=34.4,2.1 \mathrm{~Hz}), 115.42(\mathrm{dd}, J=23.9,1.7 \mathrm{~Hz})$, $86.14(\mathrm{dd}, J=184.9,8.4 \mathrm{~Hz}), 66.39$ (dd, $J=25.6,4.0 \mathrm{~Hz}), 44.82,22.44$ (d, $J=23.9 \mathrm{~Hz}), 22.33$ (d, $J=24.3 \mathrm{~Hz}), 18.21$ (d, $J=8.1 \mathrm{~Hz}$ ).

${ }^{19} \mathrm{~F}$ NMR (376 MHz, $\mathrm{CDCl}_{3}$ ): $\delta=-112.46$ to $-121.80(\mathrm{~m}),-148.93$ to $154.51(\mathrm{~m})$.

HRMS (ESI): $m / z\left[\mathrm{M}+\mathrm{Na}^{+}\right]$calcd for $\mathrm{C}_{19} \mathrm{H}_{17} \mathrm{~F}_{2} \mathrm{NO}_{2} \mathrm{Na}^{+}$: 352.1120; found: 352.1130 .

\section{2-Cyano-2-fluoropropyl 2-Bromobenzoate (18)}

Prepared by following GP using $\mathbf{1 8 a}(129 \mathrm{mg}, 0.5 \mathrm{mmol})$ as substrate. Purification by flash column chromatography (silica gel, PE:EtOAc 30:1); colorless oil; yield: $72.7 \mathrm{mg}(51 \%)$.

${ }^{1} \mathrm{H}$ NMR (500 MHz, $\mathrm{CDCl}_{3}$ ): $\delta=7.91$ (dd, $J=7.1,2.3 \mathrm{~Hz}, 1 \mathrm{H}$ ), 7.76-7.65 (m, $1 \mathrm{H}), 7.48-7.33$ (m, $2 \mathrm{H}), 4.78-4.24(\mathrm{~m}, 2 \mathrm{H}), 1.88$ (d, J = $20.9 \mathrm{~Hz}, 3$ $\mathrm{H})$.

${ }^{13} \mathrm{C}$ NMR $\left(126 \mathrm{MHz}, \mathrm{CDCl}_{3}\right): \delta=164.45,134.60,133.37,131.80$, $130.03,127.29,122.20,116.07(\mathrm{~d}, J=34.1 \mathrm{~Hz}), 85.84(\mathrm{~d}, J=184.9 \mathrm{~Hz})$, $66.79(\mathrm{~d}, J=25.9 \mathrm{~Hz}), 22.43(\mathrm{~d}, J=23.9 \mathrm{~Hz})$.

${ }^{19} \mathrm{~F}$ NMR (471 MHz, $\left.\mathrm{CDCl}_{3}\right): \delta=-152.34(\mathrm{pd}, J=20.9,14.7 \mathrm{~Hz}$ ).

HRMS (ESI): $m / z\left[\mathrm{M}+\mathrm{H}^{+}\right]$calcd for $\mathrm{C}_{12} \mathrm{H}_{9} \mathrm{BrFNO}_{2}{ }^{+}$: 285.9873; found: 285.9876 .
2-Cyano-2-fluoropropyl 5-(2,5-Dimethylphenoxy)-2,2-dimethylpentanoate (19)

Prepared by following GP using 19a (30.8 mg, $0.1 \mathrm{mmol}$ ) as substrate. Purification by flash column chromatography (silica gel, PE:EtOAc 20:1); colorless oil; yield: $18.1 \mathrm{mg}(54 \%)$.

${ }^{1} \mathrm{H}$ NMR $\left(400 \mathrm{MHz}, \mathrm{CDCl}_{3}\right): \delta=7.00(\mathrm{~d}, J=7.5 \mathrm{~Hz}, 1 \mathrm{H}), 6.66(\mathrm{~d}, J=7.3$ $\mathrm{Hz}, 1 \mathrm{H}), 6.60$ (s, $1 \mathrm{H}), 4.48-4.21$ (m, $2 \mathrm{H}), 4.00-3.85$ (m, $2 \mathrm{H}), 2.30$ (s, 3 $\mathrm{H}), 2.17(\mathrm{~s}, 3 \mathrm{H}), 1.84-1.70(\mathrm{~m}, 7 \mathrm{H}), 1.28(\mathrm{~s}, 6 \mathrm{H})$.

${ }^{13} \mathrm{C}$ NMR $\left(101 \mathrm{MHz}, \mathrm{CDCl}_{3}\right): \delta=176.53,156.85,136.48,130.29$, $123.57,120.71,116.18$ (d, $J=34.5 \mathrm{~Hz}), 111.92,86.18(\mathrm{~d}, J=184.5 \mathrm{~Hz})$, $67.69,66.00(\mathrm{~d}, J=25.5 \mathrm{~Hz}), 42.34,36.94,25.10,25.02,22.40$ (d, $J=$ $24.0 \mathrm{~Hz}), 21.42,15.78$.

${ }^{19} \mathrm{~F} \mathrm{NMR} \mathrm{(376} \mathrm{MHz,} \mathrm{CDCl}_{3}$ ): $\delta=-146.08$ to $-162.75(\mathrm{~m})$. HRMS (ESI): $\mathrm{m} / \mathrm{z}\left[\mathrm{M}+\mathrm{H}^{+}\right.$] calcd for $\mathrm{C}_{19} \mathrm{H}_{27} \mathrm{FNO}_{3}{ }^{+}$: 336.1969; found: 336.1971 .

\section{2-Cyano-2-fluoropropyl 2-\{4-[(2-0xocyclopentyl)methyl]phe- nyl\}propanoate (20)}

Prepared by following GP using $\mathbf{2 0 a}$ (30.4 mg, $0.1 \mathrm{mmol}$ ) as substrate. Purification by flash column chromatography (silica gel, PE:EtOAc 20:1); colorless oil; yield: $12.9 \mathrm{mg}(39 \%) ; \mathrm{dr}=1: 1: 1.6$.

${ }^{1} \mathrm{H}$ NMR $\left(500 \mathrm{MHz}, \mathrm{CDCl}_{3}\right): \delta=7.23(\mathrm{~d}, J=7.8 \mathrm{~Hz}, 2 \mathrm{H}), 7.13(\mathrm{~d}, J=7.8$ $\mathrm{Hz}, 2 \mathrm{H}$ ), 4.32 (ddt, $J=26.4,20.2,13.8 \mathrm{~Hz}, 2 \mathrm{H}), 3.80(\mathrm{q}, J=7.1 \mathrm{~Hz}, 1 \mathrm{H}$ ), $3.12(\mathrm{dd}, J=13.9,3.9 \mathrm{~Hz}, 1 \mathrm{H}), 2.51$ (dd, $J=13.8,9.6 \mathrm{~Hz}, 1 \mathrm{H}), 2.33$ (dd, $J=17.3,7.5 \mathrm{~Hz}, 2 \mathrm{H}), 2.13-2.04(\mathrm{~m}, 2 \mathrm{H}), 2.02-1.89$ (m, $1 \mathrm{H}), 1.78-1.70$ $(\mathrm{m}, 1 \mathrm{H}), 1.66(\mathrm{~d}, J=21.1 \mathrm{~Hz}, 3 \mathrm{H}), 1.54(\mathrm{~d}, J=7.1 \mathrm{~Hz}, 3 \mathrm{H}), 1.57-1.49$ $(\mathrm{m}, 1 \mathrm{H})$

${ }^{13} \mathrm{C}$ NMR $\left(126 \mathrm{MHz}, \mathrm{CDCl}_{3}\right): \delta=220.28,173.50(\mathrm{~d}, J=5.0 \mathrm{~Hz}), 139.45$, $137.39,129.41,127.74,116.20(\mathrm{~d}, J=32.4 \mathrm{~Hz}), 86.16$ (dd, $J=184.8,9.1$ $\mathrm{Hz}$ ), 66.15 (dd, $J=26.2,2.3 \mathrm{~Hz}$ ), 51.08, 44.96, 38.32, 35.31, 29.28, $22.37(\mathrm{dd}, J=24.0,15.7 \mathrm{~Hz}), 20.68,18.22(\mathrm{dd}, J=8.6,2.3 \mathrm{~Hz})$.

${ }^{19} \mathrm{~F}$ NMR (376 MHz, $\mathrm{CDCl}_{3}$ ): $\delta=-147.81$ to $-162.94(\mathrm{~m})$.

HRMS (ESI): $m / z\left[\mathrm{M}+\mathrm{H}^{+}\right]$calcd for $\mathrm{C}_{19} \mathrm{H}_{23} \mathrm{FNO}_{3}{ }^{+}$: 332.1656; found: 332.1664 .

\section{Conflict of Interest}

The authors declare no conflict of interest.

\section{Funding Information}

We gratefully acknowledge the National Science Foundation of China (21971228, 21772187) for financial support.

\section{Supporting Information}

Supporting information for this article is available online at https://doi.org/10.1055/s-0040-1719841.

\section{References}

(1) Chen, P.; Liu, G. Eur. J. Org. Chem. 2015, 4295.

(2) (a) Purser, S.; Moore, R. P.; Swallow, S.; Gouverneur, V. Chem. Soc. Rev. 2008, 37, 320. (b) Ni, C.; Hu, J. Chem. Soc. Rev. 2016, 45 , 5441. (c) Zhou, Y.; Wang, J.; Gu, Z.; Wang, S.; Zhu, W.; Aceña, J. L.; Soloshonok, V. A.; Izawa, K.; Liu, H. Chem. Rev. 2016, 116, 
422. (d) Zhu, Y.; Wang, J.; Shibata, N.; Sodeoka, M.; Soloshonok, V. A.; Coelho, J. A. S.; Toste, F. D. Chem. Rev. 2018, 118, 3887. (e) Jeschke, P. ChemBioChem 2004, 5, 570.

(3) (a) Murray, T. K.; Whalley, K.; Robinson, C. S.; Ward, M. A.; Hicks, C. A.; Caroline, A.; Lodge, D.; Vandergriff, J. L.; Baumbarger, P.; Siuda, E.; Gates, M.; Ogden, A. M. J. Pharmacol. Exp. Ther. 2003, 306, 752. (b) Cox, C. D.; Coleman, P. J.; Breslin, M. J.; Whitman, D. B.; Garbaccio, R. M.; Fraley, M. E.; Buser, C. A.; Walsh, E. S.; Hamilton, K.; Schaber, M. D.; Lobell, R. B.; Tao, W.; Davide, J. P.; Diehl, R. E.; Abrams, M. T.; South, V. J.; Huber, H. E.; Torrent, M.; Prueksaritanont, T.; Li, C.; Slaughter, D. E.; Mahan, E.; Fernandez-Metzler, C.; Yan, Y.; Kuo, L. C.; Kohl, N. E.; Hartman, G. D. J. Med. Chem. 2008, 51, 4239. (c) Qiu, J.; Silverman, R. B. J. Med. Chem. 2000, 43, 706. (d) Reichman, U.; Watanabe, K. A.; Fox, J. J. Carbohydr. Res. 1975, 42, 233. (e) Asahina, Y.; Takei, M.; Kimura, T.; Fukuda, Y. J. Med. Chem. 2008, 51, 3238 .

(4) Shewalkara, M. P.; Reddya, B. V. B.; Shinde, D. B. Lett. Org. Chem. 2015, 12, 222.

(5) (a) Shibata, N.; Suzuki, E.; Asahi, T.; Shiro, M. J. Am. Chem. Soc. 2001, 123, 7001. (b) Park, E. J.; Kim, H. R.; Joung, C. U.; Kim, D. Y. Bull. Korean Chem. Soc. 2004, 25, 1451. (c) Kim, H. R.; Kim, D. Y. Tetrahedron Lett. 2005, 46, 3115. (d) Moriya, K.; Hamashima, Y.; Sodeoka, M. Synlett 2007, 1139. (e) Kim, S. M.; Kang, Y. K.; Cho, M. J.; Mang, J. Y.; Kim, D. Y. Bull. Korean Chem. Soc. 2007, 28, 2435. (f) Kang, Y. K.; Cho, M. J.; Kim, S. M.; Kim, D. Y. Synlett 2007, 1135. (g) Kwon, B. K.; Mang, J. Y.; Kim, D. Y. Bull. Korean Chem. Soc. 2012, 33, 2481.

(6) (a) Balaji, P. V.; Brewitz, L.; Kumagai, N.; Shibasaki, M. Angew. Chem. Int. Ed. 2019, 58, 2644. (b) Ding, R.; De los Santos, Z. A.; Wolf, C. ACS Catal. 2019, 9, 2169. (c) Balaji, P. V.; Li, Z.; Saito, A.; Kumagai, N.; Shibasaki, M. Chem. Eur. J. 2020, 26, 15524. (d) Chen, D.-Y.; Song, S.; Chen, L.-Y.; Ren, X.; Li, Y. Tetrahedron Lett. 2021, 68, 152919.
(7) (a) Su, Y.-S.; Feng, G.-S.; Wang, Z.-Y.; Lan, Q.; Wang, X.-S. Angew. Chem. Int. Ed. 2015, 54, 6003. (b) Li, G.; Wang, T.; Fei, F.; Su, Y.M.; Li, Y.; Lan, Q.; Wang, X.-S. Angew. Chem. Int. Ed. 2016, 55, 3491. (c) Li, C.; Cao, Y.-X.; Wang, R.; Wang, Y.-N.; Lan, Q.; Wang, X.-S. Nat. Commun. 2018, 9, 4951. (d) Sheng, J.; Ni, H.-Q.; Zhang, H.-R.; Zhang, K.-F.; Wang, Y.-N.; Wang, X.-S. Angew. Chem. Int. Ed. 2018, 57, 7634. (e) Li, C.; Cao, Y.-X.; Jin, R.-X.; Bian, K.-J.; Qin, Z.-Y.; Lan, Q.; Wang, X.-S. Chem. Sci. 2019, 10, 9285. (f) Zhang, K.-F.; Bian, K.-J.; Li, C.; Sheng, J.; Li, Y.; Wang, X.-S. Angew. Chem. Int. Ed. 2019, 58, 5069.

(8) Liu, J.; Yuan, Q.; Toste, F. D.; Sigman, M. S. Nat. Chem. 2019, 11, 710.

(9) (a) Crossley, S. W. M.; Obradors, C.; Martinez, R. M.; Shenvi, R. A. Chem. Rev. 2016, 116, 8912. (b) Gaspar, B.; Carreira, E. M. J. Am. Chem. Soc. 2009, 131, 13214. (c) Gaspar, B.; Carreira, E. M. Angew. Chem. Int. Ed. 2007, 46, 4519. (d) Ma, X.; Herzon, S. B. J. Am. Chem. Soc. 2016, 138, 8718. (e) Lo, J. C.; Gui, J.; Yabe, Y.; Pan, C.-M.; Baran, P. S. Nature 2014, 516, 343. (f) Green, S. A.; Matos, J. L. M.; Yagi, A.; Shenvi, R. A. J. Am. Chem. Soc. 2016, 138, 12779. (g) Shevick, S. L.; Obradors, C.; Shenvi, R. A. J. Am. Chem. Soc. 2018, 140, 12056. (h) Green, S. A.; Huffman, T. R.; McCourt, R. O.; van der Puyl, V.; Shenvi, R. A. J. Am. Chem. Soc. 2019, 141, 7709. (i) Green, S. A.; Vásquez-Céspedes, S.; Shenvi, R. A. J. Am. Chem. Soc. 2018, 140, 11317. (j) Zhang, B.; He, J.; Li, Y.; Song, T.; Fang, Y.; Li, C. J. Am. Chem. Soc. 2021, 143, 4955. (k) Tokuyasu, T.; Kunikawa, S.; Masuyama, A.; Nojima, M. Org. Lett. 2002, 4, 3595. (l) Waser, J.; Nambu, H.; Carreira, E. M. J. Am. Chem. Soc. 2005 , $127,8294$.

(10) Tkachenko, A. N.; Radchenko, D. S.; Mykhailiuk, P. K.; Grygorenko, O. O.; Komaro, I. V. Org. Lett. 2009, 11, 5674.

(11) Thomson, C. J.; Zhang, Q.; Al-Maharik, N.; Bühl, M.; Cordes, D. B.; Slawin, A. M. Z.; O'Hagan, D. Chem. Commun. 2018, 54, 8415. 